\title{
Variation in leaf anatomy, vascular traits and nanomechanical cell-wall properties among European beech (Fagus sylvatica L.) provenances
}

\author{
Monika Kardošová ${ }^{1} \cdot$ Hana Husárová ${ }^{1} \cdot$ Daniel Kurjak $^{1}$ - Rastislav Lagaňa ${ }^{2} \cdot$ Miriama Šuleková $^{2}$ Veronika Uhrinová $^{1}$. \\ Dušan Gömöry ${ }^{1}$ (i) · Jaroslav Duurkovič ${ }^{1}$
}

Received: 26 February 2020 / Accepted: 16 July 2020 / Published online: 24 August 2020

(C) INRAE and Springer-Verlag France SAS, part of Springer Nature 2020

\begin{abstract}
- Key message Structural and ultrastructural traits of leaf xylem vary among European beech (Fagus sylvatica $\mathbf{L}$.) provenances in relation to climate. High tracheary element lumen area was observed in central Europe and decreased towards the range margins, while tracheary element density displayed opposite trend. Leaf anatomy and xylem cell-wall deformation were associated with climate continentality at the sites of origin.

- Context Understanding structural and physiological properties of tree populations adapted to local climate in response to climate may be of direct relevance for forestry.

- Aims Identifying patterns of heritable geographical variation and phenotypic associations of leaf-anatomical, vascular and cellwall properties in European beech, a dominant tree species of European forests.

- Methods Fifteen beech populations planted in a common-garden test were studied employing gas exchange and chlorophyll $a$ fluorescence measurements as well as light and atomic force microscopies of leaf samples.

- Results Nanomechanical properties of leaf-xylem cell walls did not show a clear geographical trend, but were correlated with the temperature seasonality of the site of origin. Populations from eastern Germany, northern Poland and the Czech Republic showed lower tracheary element lumen area and higher tracheary element density compared with the other populations, while xylem conductivity negatively correlated with latitude. Thickness of leaf parenchyma layers increased towards the East and, consequently, correlated with temperature indicators of climate continentality. No correlation between structural and physiological traits was observed.
\end{abstract}

Handling Editor: Erwin Dreyer

Contribution of the co-authors DG and JD conceived and designed the experiments and conducted statistical analyses of data; MK, HH, RL, MŠ and VU carried out microscopic analyses; DK provided physiological data; RL provided scientific advice and supervised atomic-force microscopy; MK, DG and JD wrote the first draft; all authors commented the manuscript.

\author{
Miriama Šuleková \\ miriama.sulekova@gmail.com \\ Veronika Uhrinová \\ veronika_kucerova@yahoo.it \\ Jaroslav Durkovič \\ durkovic@tuzvo.sk \\ Faculty of Forestry, Technical University in Zvolen, TG Masaryka \\ 24, 96001 Zvolen, Slovakia \\ 2 Faculty of Wood Technology, Technical University in Zvolen, TG \\ Masaryka 24, 96001 Zvolen, Slovakia
}


- Conclusion The observed trends and associations with climate indicate adaptive variation of the studied traits.

Keywords Fagus sylvatica L. · Leaf parenchyma $\cdot$ Leaf xylem · Vascular traits · Modulus of elasticity $\cdot$ Atomic force microscopy

\section{Introduction}

Tree mortality caused directly or indirectly by drought has been documented in a wide range of forest ecosystems (Bréda et al. 2006; Allen et al. 2010). The everyday experience with the last summer's weather across most of Europe as well as the predictions of global climate models (IPCC 2014) indicates that the risk of such damage will probably increase in the future. Such changes currently represent the biggest challenge for the persistence and resilience of forest ecosystems. This issue is of the utmost importance for the European forestry sector.

Proper functioning of the plant hydraulic system is crucial for plant survival under water stress. Although the mechanisms of drought stress related forest decline are still a matter of vivid discussion (cf. McDowell et al. 2008 vs. Sala et al. 2010), hydraulic failure (Choat et al. 2018) along with carbon starvation (Hartmann 2015) is considered alternate primary causes of plant mortality during natural drought events. Leaves make up between 30 and $60 \%$ of plant hydraulic resistance (Yang and Tyree 1994; Sack and Holbrook 2006; Nardini and Luglio 2014). Leaf hydraulics is thus the major bottleneck of the plant hydraulic system and is an important determinant of plant productivity (Brodribb et al. 2005; Brodribb and Feild 2010). Leaf hydraulic conductance is known to vary substantially, depending both from external influences like temperature or light (Sellin and Kupper 2007; Scoffoni et al. 2008) and internal factors such as leaf ontogenesis (Aasamaa et al. 2005).

Leaf hydraulics is largely determined by leaf morphology and anatomy. The hydraulic resistance of the leaf was found to be related to both venation architecture and mesophyll structure (Buckley et al. 2015; Fletcher et al. 2018). Moreover, properties of cell walls such as elasticity or deformation also play a role in water conductance and the capacity of a tree to cope with water deficit (Zhang et al. 2014; Nadal et al. 2018). Several studies have demonstrated that functional traits associated with plant hydraulics reflect adaptation to water supply (Sack and Frole 2006; Nardini et al. 2012; Nadal et al. 2018) and are thus expected to be under strong genetic control. However, majority of these studies focused on the interspecific variation, although on the intraspecific level adaptive variation of leaf hydraulic traits is equally plausible as that of stem traits (Stojnić et al. 2018).

The biomechanical properties of the cellular microenvironment, notably its rigidity and stiffness, possess a regulatory role for a variety of cellular responses including adhesion, migration, shape and division (Janmey et al. 2009; Dufrêne et al. 2013). In forest trees, the mechanical resistance of lignocellulosic vascular architecture to the xylem conduit cavitation events is determined by the stiffness of the cell wall that is quantified by modulus of elasticity (MOE) and deformation. The higher MOE and the lower deformation under the mechanical load, the better resistance of cell walls to the intrinsic pressure inside $\mathrm{xy}$ lem conduits under drought conditions as well as the higher overall stiffness of leaf midribs under windy and rainy conditions. Dissipation deals with a deformation resistance of a material. In atomic force microscopy (AFM), energy dissipated between the tip and the sample during each tap on the cell wall surface is related to the toughness of a material, and thus may be used to evaluate for example a degree of cell wall degradation. It was reported that biodegradation rapidly decreases xylem toughness measured by dynamic tests (Clausen 2010). Adhesive forces and interactions between neighbouring cells are essential for both cell development and tissue morphogenesis (Lommel et al. 2013; Arslan et al. 2015). In addition, changes in adhesion may be a good indicator of alterations in cell wall biopolymer distribution and orientation as well as chemical alterations in cell walls (Ďurkovič et al. 2013). Recent nanoscale cell wall imaging using the AFM technique called PeakForce quantitative nanomechanical mapping (PeakForce QNM) has proved to be a valuable tool for assessing not only a topography of lignocellulose architectural structures but also their nanomechanical properties such as MOE, adhesion, deformation and dissipation (Ren et al. 2015; Durkovič et al. 2017). Chemical composition of the cell wall, which largely determines nanomechanical properties of different structural domains, is known to be genetically determined in trees (Stackpole et al. 2011; Guerra et al. 2016), and thus may be targeted by local adaptation.

Our study focused on European beech (Fagus sylvatica L.) as the most important temperate broadleaved tree of Europe. In spite of a current expansion of beech in many regions (Poljanec et al. 2010), beech is declining at the rear edge of its distribution range due to drought stress (Rennenberg et al. 2004; Czucz et al., 2011). The objectives of this study were (i) to assess the variation of leaf anatomical traits, leaf vascular traits and nanomechanical properties of leaf xylem cell walls among different origins of beech; (ii) to identify of geographical and climatic trends in these traits; and (iii) to identify their relationships with growth and physiological traits. 


\section{Materials and methods}

\subsection{Experimental material}

The study relies on an international common-garden (provenance) experiment with European beech established in 1998 (seed sowing 1996). The Slovak trial, located at Tále near the town of Zvolen, central Slovakia $\left(48^{\circ} 38^{\prime} \mathrm{N}, 19^{\circ}\right.$ $02^{\prime}$ E, $810 \mathrm{~m}$ a.s.1.), comprises 32 provenances covering most of the distribution range of beech in Europe, out of which 15 were used in this study (Table 1). The test was established in a former forest nursery under a randomized complete block design with three blocks, each plot containing initially 50 plants planted at $2 \times 1 \mathrm{~m}$ spacing. During the period 2007-2013, several independent studies were performed here. To minimize the effects of history and demography on genetic variation underlying the studied structural, physiological and growth traits, only populations originated from the Slovenian glacial refugium (cf. Magri et al. 2006; Gömöry et al. 2007) were included in all studies.

Basic climatic variables (annual and vegetation-season temperature average and precipitation totals) of the sites of origin were taken from the database maintained by the provenance-experiment coordinator (J.H. von Thünen Institute, Grosshansdorf, Germany), and complemented by bioclimatic variables, reflecting temperature and precipitation fluctuation and thus climatic stress. The following variables were taken from the WorldClim high-resolution interpolated climate database (Hijmans et al. 2005): BIO4 (temperature seasonality), BIO5 (maximum temperature of the warmest month), BIO6 (minimum temperature of the coldest month), $\mathrm{BIO} 7$ (temperature annual range), $\mathrm{BIO} 13$ (precipitation of the wettest month), BIO14 (precipitation of the driest month) and BIO15 (precipitation seasonality). The bioclimatic variables were derived from meteorological data within the period 1960-1990 at the 30" resolution. Moreover, the Ellenberg's climatic quotient was calculated, as it is a good indicator for the distribution of beech (Czucz et al., 2011).

\subsection{Assessment of leaf traits}

Leaves for the assessment of structural leaf and vascular traits and nanomechanical cell-wall properties were collected in two blocks from four trees per provenance (two trees per block) (Ďurkovič, \& Kardošová 2020). Fully developed sun leaves (one leaf per tree) were sampled from the upper third of the crown on the northern side on July 15, 2013. Immediately after collection, a leaf segment of $5 \times 5 \mathrm{~mm}$ including the midrib was cut of the leaf base and fixed in 5\% (v/v) glutaraldehyde in a $0.1 \mathrm{M}$ cacodylate buffer at $\mathrm{pH} 7.0$, dehydrated through a gradient series of ethanol, cleared with xylene and embedded in paraffin. Cross-sections, approximately $15 \mu \mathrm{m}$ thick, were cut using a Bright Series 8000 retracting base sledge microtome (Bright Instruments, Luton, UK), deparaffinized in xylene, mounted on circle glass slides (11 mm in diameter) coated with (3-aminopropyl)triethoxysilane, and allowed to air dry in sterile Petri dishes. PeakForce QNM measurements were carried out using a MultiMode 8

Table 1 List of provenances with geographical coordinates and sample sizes used for measurements of structural and physiological traits

\begin{tabular}{|c|c|c|c|c|c|c|c|c|c|c|c|c|c|}
\hline No. & Name & Country & long & & lat & & elev & leaf & vascular & cell wall & gas & chl fluo & therm \\
\hline 3 & Picardie & FR & $3^{\circ}$ & $06^{\prime}$ & $49^{\circ}$ & $15^{\prime}$ & 140 & 4 & 4 & 3 & 4 & 4 & \\
\hline 5 & Bretagne & FR & $1^{\circ}$ & $09^{\prime}$ & $48^{\circ}$ & $22^{\prime}$ & 180 & 4 & 4 & 3 & 4 & 4 & \\
\hline 12 & Gruenewald & NL & $6^{\circ}$ & $12^{\prime}$ & $49^{\circ}$ & $40^{\prime}$ & 400 & 4 & 4 & & 4 & 4 & 4 \\
\hline 15 & Elspeet & NL & $5^{\circ}$ & $48^{\prime}$ & $52^{\circ}$ & $17^{\prime}$ & 33 & 4 & 3 & & & 4 & \\
\hline 23 & Torup & SE & $13^{\circ}$ & $12^{\prime}$ & $55^{\circ}$ & $34^{\prime}$ & 40 & 4 & 4 & & & 4 & 4 \\
\hline 26 & Farchau & $\mathrm{DE}$ & $10^{\circ}$ & $40^{\prime}$ & $53^{\circ}$ & $39^{\prime}$ & 55 & 4 & 4 & & 4 & 4 & 4 \\
\hline 30 & Belzig & $\mathrm{DE}$ & $12^{\circ}$ & $25^{\prime}$ & $52^{\circ}$ & $03^{\prime}$ & 140 & 4 & 4 & 3 & 4 & 4 & 4 \\
\hline 35 & Hinterstoder & $\mathrm{AT}$ & $14^{\circ}$ & $06^{\prime}$ & $47^{\circ}$ & $43^{\prime}$ & 1250 & 4 & 4 & 3 & 4 & 4 & 3 \\
\hline 36 & Eisenerz & $\mathrm{AT}$ & $14^{\circ}$ & $51^{\prime}$ & $47^{\circ}$ & $32^{\prime}$ & 1100 & 4 & 4 & 3 & 4 & 4 & 4 \\
\hline 39 & Jaworze & PL & $19^{\circ}$ & $10^{\prime}$ & $49^{\circ}$ & $50^{\prime}$ & 450 & 4 & 3 & & 4 & 4 & 3 \\
\hline 43 & Jawornik & PL & $22^{\circ}$ & $49^{\prime}$ & $49^{\circ}$ & $15^{\prime}$ & 900 & 4 & 4 & & 4 & 4 & 4 \\
\hline 50 & Janov-Načetín & $\mathrm{CZ}$ & $13^{\circ}$ & $15^{\prime}$ & $50^{\circ}$ & $34^{\prime}$ & 800 & 4 & 4 & 3 & & 4 & \\
\hline 55 & Postojna Javor & SI & $14^{\circ}$ & $21^{\prime}$ & $45^{\circ}$ & $44^{\prime}$ & 1040 & 4 & 4 & 3 & 3 & 3 & \\
\hline 62 & Aarberg & $\mathrm{CH}$ & $7^{\circ}$ & $15^{\prime}$ & $47^{\circ}$ & $02^{\prime}$ & 530 & 4 & 4 & & & 3 & \\
\hline 67 & Bilowo & PL & $18^{\circ}$ & $10^{\prime}$ & $54^{\circ}$ & $20^{\prime}$ & 250 & 4 & 4 & 3 & & 4 & 4 \\
\hline
\end{tabular}

Growth and phenology were scored exhaustively

long, longitude; lat, latitude; elev, elevation (m a.s.1.); leaf, leaf structural traits; vascular, vascular traits; cell wall, nanomechanical properties of cell walls; gas, gasometry; chl fluo, chlorophyll a fluorescence; therm, PSII thermostability 
atomic force microscope with a Nanoscope V controller (Bruker Nano Surfaces, Santa Barbara, CA, USA). Cell walls of tracheary elements were tapped by a silicon cantilever MPP-13120, model TAP525A (Bruker AFM Probes, Camarillo, CA, USA) with a spring constant of $139 \mathrm{~N} \mathrm{~m}^{-1}$, deflection sensitivity of $59.9 \mathrm{~nm} \mathrm{~V}^{-1}$, and resonance frequency of $522.2 \mathrm{kHz}$, at $25{ }^{\circ} \mathrm{C}$ and ambient air pressure. Measurements of the reduced Young's modulus of elasticity (MOE), adhesion, deformation and dissipation were performed at low approach tip velocities of $0.64 \mu \mathrm{m} \mathrm{s}{ }^{-1}$. The raw data of MOE were imported into the MATLAB software, version 7 (MathWorks, Natick, MA, USA), and the height gradient was calculated for each image pixel. Values corresponding to 'steep' points, where surface slope exceeded more than 20 degrees, were not used (Ďurkovič et al., 2017). AFM imaging of the cell wall surfaces of tracheary elements in leaf midribs coming from two contrasting provenances, is presented in Fig. 1.
Vascular characteristics of the leaf midrib primary xylem such as tracheary element lumen area $(A)$ and tracheary element densities $(N)$ per $0.1 \mathrm{~mm}^{2}$ of the primary xylem area, along with the thickness of the leaf, mesophyll, palisade and spongy parenchyma, were determined on the cross-sections, approximately $10 \mu \mathrm{m}$ thick. The cross-sections were stained with $1 \%$ safranin in $50 \%$ ethanol for $1 \mathrm{~min}$, followed by $1 \%$ Alcian blue in $0.05 \%$ acetic acid containing $0.04 \%$ formaldehyde, rinsed with water, dehydrated in an ascending ethanol series, rinsed with xylene, mounted in a drop of Canada balsam mounting medium beneath a coverslip and examined immediately with an Olympus BX50F microscope (Olympus Europa, Hamburg, Germany). The quantitative data were measured using the NIS-Elements AR 3.0 image analysis software (Laboratory Imaging, Prague, Czech Republic). The additional indicators of vascular strategy such as tracheary element lumen fraction $(F=A \times N)$ and the tracheary element size-to-number ratio $(S=A / N)$ were calculated as described

\section{PROVENANCE 30 BELZIG}

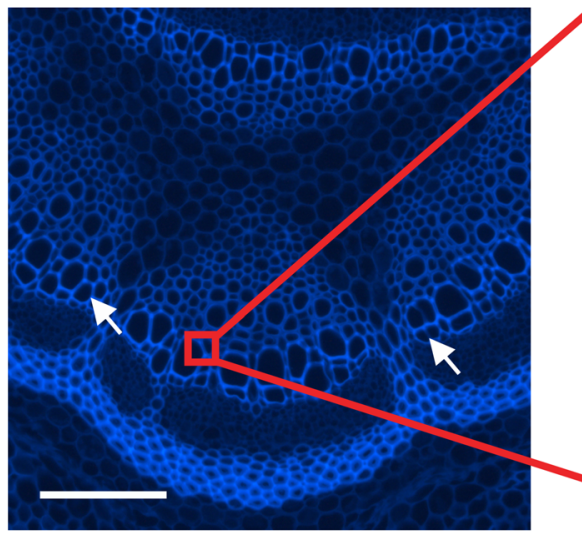

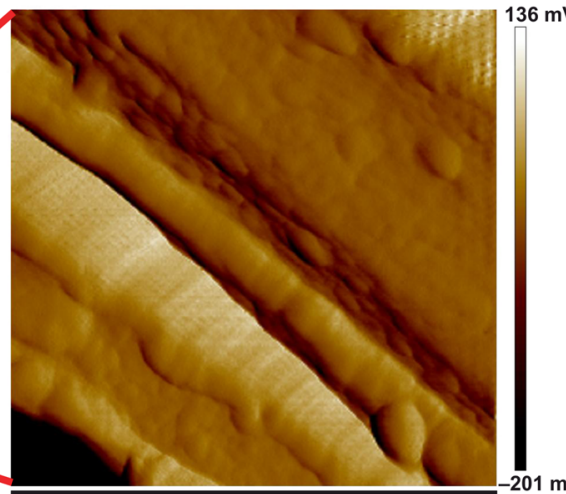

Peak Force Error

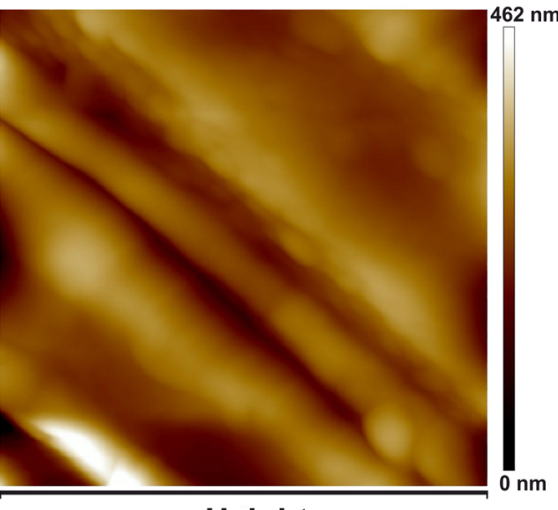

Height

\section{PROVENANCE 55 POSTOJNA JAVOR}

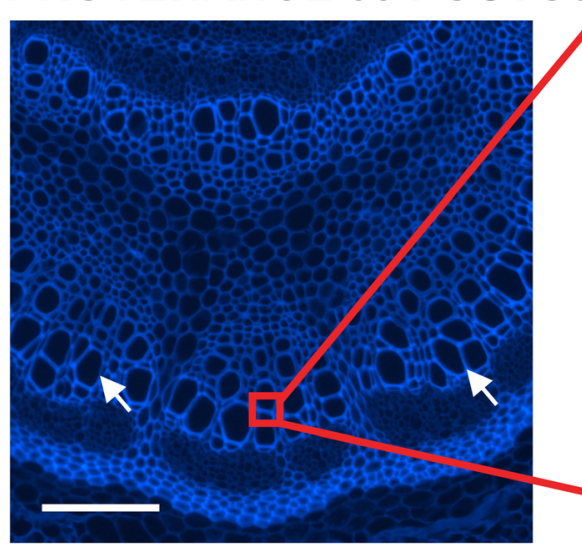

Fig. 1 Lignin autofluorescence images of primary xylem in the leaf midrib (left images), atomic force microscopy (AFM) peak force error images (middle images) and AFM flatten height images (right images) of neighbouring cell wall surfaces of the tracheary elements. Scale bars for the provenance 30 sample: $50 \mu \mathrm{m}$ for the fluorescence microscopy image, and $1.5 \mu \mathrm{m}$ for AFM images. Scale bars for the provenance 55 sample:

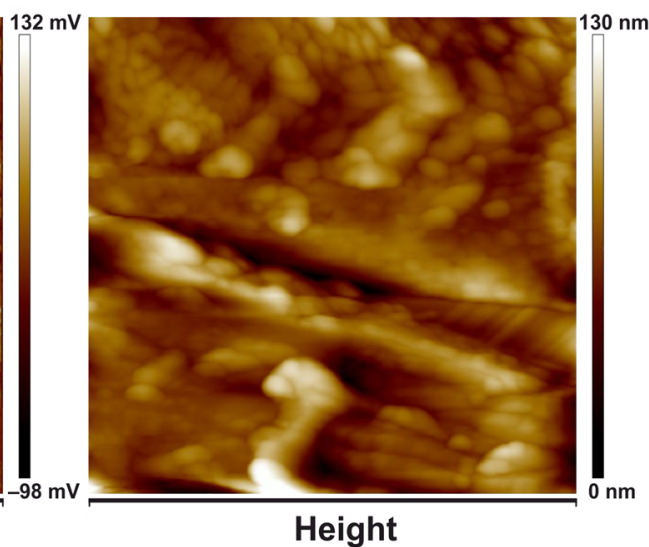

Height

$50 \mu \mathrm{m}$ for the fluorescence microscopy image, and $1.9 \mu \mathrm{m}$ for AFM images. Arrows show contrasts in sizes of tracheary element lumen areas. Tracheary elements with larger lumen areas (typical for the provenance 55) support a fast and efficient water transport to leaf tissues, whereas tracheary elements with smaller lumen areas (typical for the provenance 30) indicate a safety against embolism in drought-affected periods 
in Zanne et al. (2010). Total theoretical relative conductivity (RC) per $0.1 \mathrm{~mm}^{2}$ of the primary xylem area was calculated as the sum of individual RCs divided by the area of a crosssection of primary xylem, while the individual RC was calculated according to Zimmermann (1983) as the fourth power of the equivalent circle diameter of the tracheary element lumen.

Growth traits (height, diameter at breast height), vegetative phenology (timing of budburst and leaf discolouration) and late-frost damage were assessed exhaustively in 2007. Details of the assessment of budburst and leaf-discolouration phenology (scoring scales, scoring dates, estimation procedure for the Julian mid-days of spring flushing and autumn discolouration, respectively) and frost-damage scoring are given in Gömöry and Paule (2011). For the purposes of this study, only data of the trees scored for leaf structural properties were used.

Photosynthetic parameters were measured during the 2013 and 2014 summer seasons on the same trees as those that were used for the assessment of structural and nanomechanical traits (cf. Gömöry et al. 2015). The following physiological variables were assessed:

(i) Fast kinetics of chlorophyll a fluorescence: the Plant Efficiency Analyzer (Handy PEA, Hansatech, Kings Lynn, UK) was used to measure the maximum fluorescence yield $\left(F_{v} / F_{m}\right)$, photosynthetic performance index $(P I)$, and density of active PS II reaction centers $(R C /$ $A B S$ ) on sun exposed branches from upper third of the crown; to avoid the effect of weather and daily dynamics, all measurements were done on leaves kept in water bath at a standard temperature of $30{ }^{\circ} \mathrm{C}$ for $30 \mathrm{~min}$. After a 30 min dark adaptation, the samples were irradiated using a saturation pulse of $3500 \mu \mathrm{mol} \mathrm{m} \mathrm{s}^{-2} . F_{v} / F_{m}$ was calculated as the ratio of the maximal variable fluorescence $F_{v}$ and the maximum fluorescence emission $F_{m}, P I$ and $R C$ / $A B S$ were calculated according to Strasser et al. (2000) and Brestič and Živčák (2013). Data were analyzed using the Biolyzer software (Laboratory of Bioenergetics, University of Geneva, Switzerland).

(ii) Thermal stability of photosystem II: water bath WNE22 (Memmert, Germany) was used to simulate different temperatures ranging from $30{ }^{\circ} \mathrm{C}$ up to $48{ }^{\circ} \mathrm{C}$ (step $3{ }^{\circ} \mathrm{C}$ ). Leaves were kept in enclosed Erlenmeyer flasks and put into water bath at the respective temperature for $30 \mathrm{~min}$. The thermal sensitivity of PSII was assessed by $T_{85}$, the temperature at which $F_{v} / F_{m}$ declines to $85 \%$ of the maximum value (Froux et al. 2004).

(iii) Gas exchange: gasometric system Li-6400XT equipped with a 6400-02B LED light source (LI-COR Biosciences, Lincoln, USA) was used for measurements in situ. The $\mathrm{CO}_{2}$ concentration in the chamber was kept at $385 \mu \mathrm{mol} \mathrm{mol}{ }^{-1}$, the saturating PAR was maintained at $1300 \mu \mathrm{mol} \mathrm{m} \mathrm{m}^{-2} \mathrm{~s}^{-1}$, the air temperature in the chamber was $20.5 \pm 0.5{ }^{\circ} \mathrm{C} . \mathrm{CO}_{2}$ assimilation, stomatal conductance, transpiration rate and the instantaneous water-use efficiency (WUE) were evaluated.

As the measurements of different structural and nanomechanical traits are differently labour-intensive, sample sizes varied accordingly, both in terms of number of populations and number of trees per population. An overview of the studied traits including the provenances used and sample sizes is given in Table 1.

\subsection{Data treatment}

Because of inhomogeneity of variances and non-normal distributions of residuals, differences in structural and ultrastructural traits among populations were tested using nonparametric Kruskal-Wallis test; pairwise contrasts were subsequently tested by Wilcoxon's tests (procedure NPAR1WAY; SAS 2010). Correlations of leaf structural and vascular traits and/or nanomechanical properties with growth and/or physiological traits were assessed on the individual (tree) level, while correlations with the climate of origin on the population (provenance) level; in the latter case, provenance means were correlated with climate parameters. In both cases, the procedure CORR (SAS 2010) was used. To account for potential non-linear relationships, 1st- and 2ndorder polynomial regression models were fitted using the procedure REG and were compared by adjusted $R^{2}$ and the Akaike information criterion (SAS 2010).

\section{Results}

As expected, leaf structural traits were strongly intercorrelated both on the tree level and the provenance level (Table 2). This means that individual tissue layers represent a relatively stable proportion of the total leaf thickness in all provenances. For midrib vascular traits, such evaluation makes less sense as they are mostly mathematically related to each other. Among the nanomechanical properties, modulus of elasticity was expectedly found to be negatively correlated with deformation, while adhesion was positively correlated with energy dissipation.

Nanomechanical properties (adhesion and MOE) showed marginally significant $(P<0.10)$ variation among provenances (Table 3 and Table 4), but they do not show any clear geographical trend. However, even if such a trend existed, it would hardly be discernible with only 8 provenances. Frequently even pairs of spatially and ecologically proximate populations (such as the Austrian or the French ones) show substantial differences (Fig. 2).

A kind of geographical pattern in several leaf structure and vascular traits can be observed. For illustration, geographical 
Table 2 Individual-level and provenance-level correlations between leaf structural and vascular traits, nanomechanical cell-wall properties, growth, phenological and physiological traits, geographical coordinates, climatic and bioclimatic variables

Individual-level correlations

Nanomechanical properties of xylem cell walls

\begin{tabular}{|c|c|c|c|c|c|c|}
\hline & $M O E$ & Adhes & Deform & Dissip & & $M O E$ \\
\hline \multirow[t]{2}{*}{ Adhes } & 0.2067 & & & & Adhes & 0.4302 \\
\hline & ns & & & & & ns \\
\hline Deform & $\begin{array}{l}-0.5788 \\
* *\end{array}$ & $\begin{array}{l}0.0702 \\
\text { ns }\end{array}$ & & & Deform & $\begin{array}{l}-0.5756 \\
\mathrm{~ns}\end{array}$ \\
\hline Dissip & $\begin{array}{l}0.1546 \\
\mathrm{~ns}\end{array}$ & $\begin{array}{l}0.7492 \\
* * *\end{array}$ & $\begin{array}{l}0.1072 \\
\mathrm{~ns}\end{array}$ & & Dissip & $\begin{array}{l}0.0188 \\
n s\end{array}$ \\
\hline A & 0.1198 & -0.1674 & 0.0295 & -0.0734 & $A$ & -0.0477 \\
\hline \multirow[t]{2}{*}{$N$} & $\begin{array}{l}\text { ns } \\
-0.0216\end{array}$ & $\begin{array}{l}\text { ns } \\
0.1266\end{array}$ & $\begin{array}{l}\text { ns } \\
-0.0079\end{array}$ & $\begin{array}{l}\text { ns } \\
0.1551\end{array}$ & $N$ & $\begin{array}{l}\text { ns } \\
-0.0588\end{array}$ \\
\hline & ns & ns & ns & ns & & ns \\
\hline$F$ & 0.3653 & 0.0005 & -0.1098 & 0.2461 & $F$ & -0.1305 \\
\hline$S$ & $\begin{array}{l}\text { ns } \\
-0.0185\end{array}$ & $\begin{array}{l}\text { ns } \\
-0.1766\end{array}$ & $\begin{array}{l}\mathrm{ns} \\
0.0664\end{array}$ & $\begin{array}{l}\text { ns } \\
-0.1338\end{array}$ & $S$ & $\begin{array}{l}\text { ns } \\
-0.0807\end{array}$ \\
\hline cond & $\begin{array}{l}\text { ns } \\
0.3741 \\
\text { ns }\end{array}$ & $\begin{array}{l}\text { ns } \\
-0.1242 \\
\text { ns }\end{array}$ & $\begin{array}{l}\text { ns } \\
-0.1182 \\
\text { ns }\end{array}$ & $\begin{array}{l}\mathrm{ns} \\
0.0155 \\
\mathrm{~ns}\end{array}$ & cond & $\begin{array}{l}\mathrm{ns} \\
0.0549 \\
\mathrm{~ns}\end{array}$ \\
\hline leaf & $\begin{array}{l}0.3067 \\
\text { ns }\end{array}$ & $\begin{array}{l}-0.1106 \\
\text { ns }\end{array}$ & $\begin{array}{l}-0.0846 \\
\text { ns }\end{array}$ & $\begin{array}{l}-0.2278 \\
\text { ns }\end{array}$ & leaf & $\begin{array}{l}0.2679 \\
\text { ns }\end{array}$ \\
\hline mesoph & $\begin{array}{l}0.3295 \\
\mathrm{~ns}\end{array}$ & $\begin{array}{l}-0.0921 \\
\text { ns }\end{array}$ & $\begin{array}{l}-0.0995 \\
\text { ns }\end{array}$ & $\begin{array}{l}-0.2050 \\
\mathrm{~ns}\end{array}$ & mesoph & $\begin{array}{l}0.3069 \\
\mathrm{~ns}\end{array}$ \\
\hline palisade & $\begin{array}{l}0.3203 \\
\text { ns }\end{array}$ & $\begin{array}{l}-0.0239 \\
\mathrm{~ns}\end{array}$ & $\begin{array}{l}-0.0545 \\
\text { ns }\end{array}$ & $\begin{array}{l}-0.1834 \\
\mathrm{~ns}\end{array}$ & palisade & $\begin{array}{l}0.4785 \\
\mathrm{~ns}\end{array}$ \\
\hline spongy & $\begin{array}{l}0.2478 \\
\mathrm{~ns}\end{array}$ & $\begin{array}{l}-0.1490 \\
\text { ns }\end{array}$ & $\begin{array}{l}-0.1260 \\
\text { ns }\end{array}$ & $\begin{array}{l}-0.1734 \\
\mathrm{~ns}\end{array}$ & spongy & $\begin{array}{l}0.0243 \\
\mathrm{~ns}\end{array}$ \\
\hline height & $\begin{array}{l}-0.4551 \\
*\end{array}$ & $\begin{array}{l}0.0714 \\
\text { ns }\end{array}$ & $\begin{array}{l}0.2602 \\
\mathrm{~ns}\end{array}$ & $\begin{array}{l}0.0783 \\
\mathrm{~ns}\end{array}$ & long & $\begin{array}{l}0.6178 \\
\mathrm{~ns}\end{array}$ \\
\hline$B H D$ & $\begin{array}{l}-0.4601 \\
*\end{array}$ & $\begin{array}{l}-0.0139 \\
\mathrm{~ns}\end{array}$ & $\begin{array}{l}0.3310 \\
\mathrm{~ns}\end{array}$ & $\begin{array}{l}-0.0478 \\
\mathrm{~ns}\end{array}$ & lat & $\begin{array}{l}0.3347 \\
\mathrm{~ns}\end{array}$ \\
\hline budburst & $\begin{array}{l}0.6312 \\
* *\end{array}$ & $\begin{array}{l}0.2036 \\
\mathrm{~ns}\end{array}$ & $\begin{array}{l}-0.4151 \\
*\end{array}$ & $\begin{array}{l}0.0658 \\
\mathrm{~ns}\end{array}$ & elev & $\begin{array}{l}0.2179 \\
\mathrm{~ns}\end{array}$ \\
\hline discolor & $\begin{array}{l}-0.2388 \\
\text { ns }\end{array}$ & $\begin{array}{l}-0.3528 \\
\text { ns }\end{array}$ & $\begin{array}{l}0.0618 \\
\mathrm{~ns}\end{array}$ & $\begin{array}{l}-0.3161 \\
\mathrm{~ns}\end{array}$ & $t_{a v g}$ & $\begin{array}{l}-0.5209 \\
\mathrm{~ns}\end{array}$ \\
\hline frost & $\begin{array}{l}-0.5394 \\
* *\end{array}$ & $\begin{array}{l}-0.2481 \\
\mathrm{~ns}\end{array}$ & $\begin{array}{l}0.2201 \\
\mathrm{~ns}\end{array}$ & $\begin{array}{l}0.0005 \\
\text { ns }\end{array}$ & $t_{59}$ & $\begin{array}{l}-0.4525 \\
\mathrm{~ns}\end{array}$ \\
\hline $\mathrm{CO}_{2}$ assi & $\begin{array}{l}-0.0035 \\
\text { ns }\end{array}$ & $\begin{array}{l}0.1187 \\
\text { ns }\end{array}$ & $\begin{array}{l}0.1985 \\
\text { ns }\end{array}$ & $\begin{array}{l}-0.0717 \\
\mathrm{~ns}\end{array}$ & $p_{\text {avg }}$ & $\begin{array}{l}0.2876 \\
\mathrm{~ns}\end{array}$ \\
\hline stcond & $\begin{array}{l}0.0044 \\
\mathrm{~ns}\end{array}$ & $\begin{array}{l}-0.0490 \\
\text { ns }\end{array}$ & $\begin{array}{l}0.2200 \\
\mathrm{~ns}\end{array}$ & $\begin{array}{l}0.0824 \\
\text { ns }\end{array}$ & $p_{59}$ & $\begin{array}{l}0.4046 \\
\mathrm{~ns}\end{array}$ \\
\hline transpir & $\begin{array}{l}-0.1503 \\
\mathrm{~ns}\end{array}$ & $\begin{array}{l}-0.1129 \\
\text { ns }\end{array}$ & $\begin{array}{l}0.3106 \\
\mathrm{~ns}\end{array}$ & $\begin{array}{l}0.0017 \\
\mathrm{~ns}\end{array}$ & BIO4 & $\begin{array}{l}0.6059 \\
\mathrm{~ns}\end{array}$ \\
\hline$W U E$ & $\begin{array}{l}0.0646 \\
\text { ns }\end{array}$ & $\begin{array}{l}0.2958 \\
\text { ns }\end{array}$ & $\begin{array}{l}-0.2118 \\
\text { ns }\end{array}$ & $\begin{array}{l}0.0438 \\
\text { ns }\end{array}$ & BIO5 & $\begin{array}{l}-0.4746 \\
\mathrm{~ns}\end{array}$ \\
\hline$F_{v} / F_{m}$ & $\begin{array}{l}-0.2357 \\
\mathrm{~ns}\end{array}$ & $\begin{array}{l}-0.1676 \\
\text { ns }\end{array}$ & $\begin{array}{l}0.2816 \\
\mathrm{~ns}\end{array}$ & $\begin{array}{l}0.0679 \\
\text { ns }\end{array}$ & BIO7 & $\begin{array}{l}0.5626 \\
\mathrm{~ns}\end{array}$ \\
\hline$R C / A B S$ & $\begin{array}{l}0.1672 \\
\text { ns }\end{array}$ & $\begin{array}{l}-0.1107 \\
\mathrm{~ns}\end{array}$ & $\begin{array}{l}0.1097 \\
\mathrm{~ns}\end{array}$ & $\begin{array}{l}0.0307 \\
\mathrm{~ns}\end{array}$ & BIO13 & $\begin{array}{l}0.4020 \\
\mathrm{~ns}\end{array}$ \\
\hline$P I$ & $\begin{array}{l}-0.0621 \\
\text { ns }\end{array}$ & $\begin{array}{l}-0.0830 \\
\text { ns }\end{array}$ & $\begin{array}{l}0.1689 \\
n s\end{array}$ & $\begin{array}{l}0.2334 \\
\text { ns }\end{array}$ & BIO14 & $\begin{array}{l}0.1132 \\
\mathrm{~ns}\end{array}$ \\
\hline \multirow[t]{2}{*}{$T_{85}$} & $\begin{array}{l}-0.1233 \\
\mathrm{~ns}\end{array}$ & $\begin{array}{l}-0.3183 \\
\mathrm{~ns}\end{array}$ & $\begin{array}{l}0.0971 \\
\mathrm{~ns}\end{array}$ & $\begin{array}{l}-0.0826 \\
\mathrm{~ns}\end{array}$ & BIO15 & $\begin{array}{l}0.6142 \\
\mathrm{~ns}\end{array}$ \\
\hline & & & & & $E Q$ & $\begin{array}{l}0.5637 \\
\mathrm{~ns}\end{array}$ \\
\hline
\end{tabular}

Vascular traits

\begin{tabular}{|c|c|c|}
\hline$N$ & $\begin{array}{l}A \\
-0.8046 \\
* * *\end{array}$ & $N$ \\
\hline$F$ & $\begin{array}{l}0.0953 \\
\text { ns }\end{array}$ & $\begin{array}{l}0.3821 \\
* *\end{array}$ \\
\hline$S$ & $\begin{array}{l}0.9223 \\
* * *\end{array}$ & $\begin{array}{l}-0.7599 \\
* * *\end{array}$ \\
\hline cond & $\begin{array}{l}0.7267 \\
* * *\end{array}$ & $\begin{array}{l}-0.3988 \\
* *\end{array}$ \\
\hline leaf & 0.2695 & -0.3023 \\
\hline
\end{tabular}

Provenance-level correlations

$-0.0477$

$-0.0588$

$-0.1305$

$-0.0807$

.0549

.2679

0.3069

.4785

.0243

6178

.3347

.2179

$-0.5209$

$-0.4525$

.2876

0.4046

0.6059

$-0.4746$

0.5626

0.4020

0.1132

6142

0.5637

F S

cond

$\begin{array}{ll} & A \\ N & -0.6124 \\ & * \\ F & 0.4722 \\ & \text { ns } \\ S & 0.8539 \\ & * * * \\ \text { cond } & 0.8059 \\ & * * * \\ \text { leaf } & 0.3673\end{array}$

$-0.1682$

ns

$\begin{array}{ll}0.6185 & 0.4831 \\ * * * & * * *\end{array}$

$-0.1173$

0.2543

0.0912
Adhes Deform Dissip

$\begin{array}{lll}-0.1300 & & \\ \mathrm{~ns} & & \\ 0.8785 & 0.0454 & \\ * * & \mathrm{~ns} & \\ -0.2092 & -0.0626 & -0.3600 \\ \mathrm{~ns} & \mathrm{~ns} & \mathrm{~ns} \\ -0.1784 & 0.0594 & -0.1076 \\ \mathrm{~ns} & \mathrm{~ns} & \mathrm{~ns} \\ -0.6462 & -0.1741 & -0.7179 \\ \mathrm{~ns} & \mathrm{~ns} & * \\ -0.1832 & -0.0356 & -0.2984 \\ \mathrm{~ns} & \mathrm{~ns} & \mathrm{~ns} \\ -0.3170 & -0.3622 & -0.5036 \\ \mathrm{~ns} & \mathrm{~ns} & \mathrm{~ns}\end{array}$

$\begin{array}{lll}0.2343 & 0.0564 & 0.0381\end{array}$

ns ns ns

$\begin{array}{lll}0.2175 & 0.0080 & 0.0066\end{array}$

$\begin{array}{lll}\text { ns } & \text { ns } & \text { ns } \\ 0.1873 & 0.0517 & -0.0933\end{array}$

$\begin{array}{lll}0.1873 & 0.0517 & -0.0933 \\ \text { ns } & \text { ns } & \text { ns }\end{array}$

$\begin{array}{lll}0.2178 & -0.0512 \quad 0.1371\end{array}$

ns ns ns

$\begin{array}{lll}0.4817 & -0.7607 & 0.3146\end{array}$

ns ns ns

$\begin{array}{lll}0.6975 & -0.0492 & 0.6752\end{array}$

ns ns ns

$\begin{array}{lll}-0.2824 & -0.5246 & -0.3369\end{array}$

ns ns ns

$\begin{array}{lll}-0.2007 & 0.6434 & -0.1109\end{array}$

ns ns ns

$\begin{array}{lll}-0.1113 & 0.5603 & -0.0472\end{array}$

ns ns ns

$\begin{array}{lll}-0.3512 & -0.5033 & -0.4978\end{array}$

ns ns ns

$\begin{array}{lll}-0.1934 & -0.5721 & -0.3609\end{array}$

ns ns ns

$\begin{array}{lll}0.5599 & -0.7492 & 0.3916\end{array}$

ns $\quad * \quad$ ns

$\begin{array}{lll}-0.1429 & 0.5505 & -0.1329\end{array}$

ns ns ns

$\begin{array}{lll}0.3332 & -0.7143 \quad 0.0897\end{array}$

ns ns ns

$\begin{array}{lll}-0.1971 & -0.6117 & -0.3614\end{array}$

ns ns ns

$\begin{array}{lll}-0.5780 & -0.3549 & -0.6758\end{array}$

ns ns ns

$\begin{array}{lll}0.6049 & -0.7011 & 0.4515\end{array}$

ns ns ns

$\begin{array}{lll}0.0751 & -0.5978 & -0.0899\end{array}$

ns ns ns

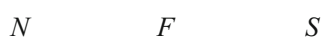

S cond

0.2365

$-0.5181 \quad 0.1159$

* ns

$\begin{array}{lll}-0.2384 & 0.8410 & 0.4805\end{array}$

ns $\quad * * * \quad$ ns

$\begin{array}{lll}-0.1621 & 0.1259 & 0.3797\end{array}$

0.1555 
Table 2 (continued)

\begin{tabular}{|c|c|c|c|c|c|c|c|c|c|c|c|}
\hline & $*$ & $*$ & ns & ns & ns & & ns & ns & ns & ns & ns \\
\hline \multirow[t]{2}{*}{ mesoph } & 0.2665 & -0.2906 & -0.1127 & 0.2556 & 0.0848 & mesoph & 0.3597 & -0.1503 & 0.1177 & 0.3862 & 0.1440 \\
\hline & $*$ & $*$ & ns & ns & ns & & ns & ns & ns & ns & ns \\
\hline \multirow[t]{2}{*}{ palisade } & 0.1012 & -0.1515 & -0.0873 & 0.0832 & -0.0053 & palisade & 0.2790 & -0.1678 & 0.0032 & 0.3225 & 0.0117 \\
\hline & ns & ns & ns & ns & ns & & ns & ns & ns & ns & ns \\
\hline \multirow[t]{2}{*}{ spongy } & 0.4064 & -0.3899 & -0.1144 & 0.4078 & 0.1776 & spongy & 0.3915 & -0.1115 & 0.2174 & 0.3970 & 0.2581 \\
\hline & $* *$ & $* *$ & ns & $* *$ & ns & & ns & ns & ns & ns & ns \\
\hline \multirow[t]{2}{*}{ height } & -0.1054 & 0.1842 & 0.0856 & -0.1044 & -0.0257 & long & 0.1164 & -0.0092 & 0.1206 & 0.0963 & 0.1319 \\
\hline & ns & ns & ns & ns & ns & & ns & ns & ns & ns & ns \\
\hline \multirow[t]{2}{*}{$B H D$} & -0.1493 & 0.2116 & -0.0110 & -0.0976 & -0.1001 & lat & -0.7005 & 0.2514 & -0.5349 & -0.5783 & -0.6988 \\
\hline & ns & ns & $\mathrm{ns}$ & ns & ns & & & ns & $*$ & $*$ & $* *$ \\
\hline \multirow[t]{2}{*}{ budburst } & -0.0163 & -0.0401 & -0.0926 & -0.0058 & -0.0819 & elev & 0.4895 & -0.2414 & 0.3036 & 0.4342 & 0.4757 \\
\hline & ns & ns & ns & ns & ns & & ns & ns & ns & ns & ns \\
\hline \multirow[t]{2}{*}{ discolor } & 0.0331 & -0.0776 & -0.2515 & 0.1466 & -0.1391 & $t_{\text {avg }}$ & 0.0192 & 0.0547 & -0.0628 & 0.1018 & -0.1300 \\
\hline & ns & ns & ns & ns & ns & & ns & ns & ns & ns & ns \\
\hline \multirow[t]{2}{*}{ frost } & -0.0227 & 0.1027 & 0.1445 & -0.0416 & 0.0795 & $t_{59}$ & 0.0832 & 0.0825 & 0.0281 & 0.1468 & -0.0583 \\
\hline & ns & ns & ns & ns & ns & & ns & ns & ns & ns & ns \\
\hline \multirow[t]{2}{*}{$\mathrm{CO}_{2}$ assi } & 0.1963 & -0.3561 & -0.1187 & 0.0904 & 0.1421 & $p_{a v g}$ & 0.4626 & -0.2693 & 0.1926 & 0.4947 & 0.4193 \\
\hline & ns & $*$ & ns & ns & ns & & ns & ns & ns & ns & ns \\
\hline \multirow[t]{2}{*}{ stcond } & 0.0997 & -0.1974 & -0.0142 & 0.0141 & 0.0875 & $p_{59}$ & 0.3879 & -0.2134 & 0.2691 & 0.3302 & 0.4398 \\
\hline & ns & ns & ns & ns & ns & & ns & $\mathrm{ns}$ & ns & ns & $\mathrm{ns}$ \\
\hline \multirow[t]{2}{*}{ transpir } & 0.1126 & -0.2013 & -0.0236 & 0.0345 & 0.1080 & BIO4 & 0.2149 & 0.0191 & 0.3401 & 0.0832 & 0.3262 \\
\hline & ns & ns & ns & ns & ns & & ns & ns & ns & ns & ns \\
\hline$W U E$ & 0.0688 & -0.1131 & -0.1317 & 0.0785 & -0.0574 & BIO5 & 0.3209 & -0.0062 & 0.3061 & 0.2505 & 0.2830 \\
\hline & ns & ns & ns & ns & ns & & ns & ns & ns & ns & ns \\
\hline$F_{v} / F_{m}$ & 0.0324 & -0.0358 & -0.0942 & 0.0647 & -0.0195 & $\mathrm{BIO7}$ & 0.4313 & -0.1143 & 0.5166 & 0.2073 & 0.5771 \\
\hline & ns & ns & ns & ns & ns & & ns & ns & $*$ & ns & $*$ \\
\hline$R C / A B S$ & 0.0883 & -0.0800 & 0.0446 & 0.0517 & 0.0729 & BIO13 & 0.4223 & -0.2148 & 0.3245 & 0.3423 & 0.4882 \\
\hline & ns & ns & $\mathrm{ns}$ & ns & ns & & ns & ns & ns & ns & ns \\
\hline$P I$ & 0.0492 & -0.0799 & -0.0586 & 0.0501 & -0.0181 & BIO14 & 0.4840 & -0.2391 & 0.1957 & 0.5476 & 0.4126 \\
\hline & ns & ns & ns & ns & ns & & ns & ns & ns & $*$ & ns \\
\hline$T_{85}$ & 0.2067 & -0.0434 & 0.2049 & 0.1387 & 0.2132 & BIO15 & 0.0547 & -0.0075 & 0.2575 & -0.1259 & 0.2240 \\
\hline & ns & ns & ns & ns & ns & & ns & $\mathrm{ns}$ & ns & ns & ns \\
\hline & & & & & & $E Q$ & 0.0833 & -0.1258 & 0.1239 & -0.0345 & 0.2490 \\
\hline & & & & & & & ns & ns & ns & ns & ns \\
\hline Leaf struc & al traits & & & & & & & & & & \\
\hline & leaf & mesoph & palisade & spongy & & leaf & & mesoph & palisade & spongy & \\
\hline mesoph & $\begin{array}{l}0.9965 \\
* * *\end{array}$ & & & & mesoph & $\begin{array}{l}0.9974 \\
* * *\end{array}$ & & & & & \\
\hline palisade & $\begin{array}{l}0.8970 \\
* * *\end{array}$ & $\begin{array}{l}0.9092 \\
* * *\end{array}$ & & & palisade & $\begin{array}{l}0.9127 \\
* * *\end{array}$ & & $\begin{array}{l}0.9315 \\
* * *\end{array}$ & & & \\
\hline spongy & 0.8546 & 0.8461 & 0.5473 & & spongy & 0.9443 & & 0.9301 & 0.7328 & & \\
\hline & $* * *$ & $* * *$ & $* * *$ & & & $* * *$ & & $* * *$ & $* *$ & & \\
\hline height & 0.0217 & 0.0224 & -0.0317 & 0.0859 & long & 0.6024 & & 0.6142 & 0.5282 & 0.6156 & \\
\hline & ns & ns & ns & ns & & $*$ & & $*$ & $*$ & $*$ & \\
\hline$B H D$ & 0.0756 & 0.0782 & 0.0396 & 0.1061 & lat & -0.0280 & & -0.0244 & 0.0399 & -0.0864 & \\
\hline & ns & ns & ns & ns & & ns & & ns & ns & ns & \\
\hline budburst & -0.1647 & -0.1508 & -0.0419 & -0.2491 & elev & 0.2478 & & 0.2485 & 0.1521 & 0.3117 & \\
\hline & ns & ns & ns & ns & & ns & & ns & ns & ns & \\
\hline discolor & 0.0125 & 0.0042 & -0.1094 & 0.1496 & $t_{\text {avg }}$ & -0.1857 & & -0.1834 & -0.0891 & -0.2535 & \\
\hline & ns & ns & ns & ns & & ns & & ns & ns & ns & \\
\hline frost & -0.1204 & -0.1349 & -0.1728 & -0.0483 & $t_{59}$ & -0.0234 & & -0.0182 & 0.0590 & -0.0939 & \\
\hline & ns & ns & ns & ns & & ns & & ns & ns & ns & \\
\hline $\mathrm{CO}_{2}$ assi & 0.2387 & 0.2132 & 0.1896 & 0.1881 & $p_{\text {avg }}$ & 0.1577 & & 0.1771 & 0.1391 & 0.1913 & \\
\hline & ns & ns & ns & ns & & ns & & ns & ns & ns & \\
\hline stcond & 0.1935 & 0.1619 & 0.1304 & 0.1613 & $p_{59}$ & 0.2489 & & 0.2647 & 0.2115 & 0.2821 & \\
\hline & ns & ns & ns & ns & & ns & & ns & ns & ns & \\
\hline transpir & 0.1819 & 0.1501 & 0.1209 & 0.1496 & $\mathrm{BIO4}$ & 0.6225 & & 0.6282 & 0.5162 & 0.6541 & \\
\hline & ns & ns & $\mathrm{ns}$ & ns & & $*$ & & $*$ & $*$ & $* *$ & \\
\hline$W U E$ & -0.0887 & -0.0731 & -0.0649 & -0.0647 & BIO5 & 0.0744 & & 0.0730 & 0.0914 & 0.0442 & \\
\hline & ns & ns & ns & ns & & ns & & ns & ns & ns & \\
\hline$F_{v} / F_{m}$ & 0.1274 & 0.1216 & 0.0630 & 0.1646 & BIO7 & 0.5818 & & 0.5821 & 0.4622 & 0.6224 & \\
\hline & ns & ns & ns & ns & & $*$ & & $*$ & ns & $*$ & \\
\hline$R C / A B S$ & 0.2310 & 0.2089 & 0.1614 & 0.2130 & BIO13 & 0.2608 & & 0.2778 & 0.2186 & 0.2994 & \\
\hline & ns & ns & ns & ns & & ns & & ns & ns & ns & \\
\hline$P I$ & 0.1178 & 0.1090 & 0.0295 & 0.1828 & BIO14 & -0.0096 & & 0.0082 & -0.0036 & 0.0195 & \\
\hline & ns & ns & ns & ns & & ns & & ns & ns & ns & \\
\hline
\end{tabular}


Table 2 (continued)

\begin{tabular}{|c|c|c|c|c|c|c|c|c|c|}
\hline$T_{85}$ & $\begin{array}{l}-0.0470 \\
\mathrm{~ns}\end{array}$ & $\begin{array}{l}-0.0548 \\
\text { ns }\end{array}$ & $\begin{array}{l}-0.1212 \\
\text { ns }\end{array}$ & $\begin{array}{l}0.0617 \\
\text { ns }\end{array}$ & $\begin{array}{l}B I O 15 \\
E Q\end{array}$ & $\begin{array}{l}0.4542 \\
\text { ns } \\
0.0902 \\
\text { ns }\end{array}$ & $\begin{array}{l}0.4609 \\
\text { ns } \\
0.0870 \\
\text { ns }\end{array}$ & $\begin{array}{l}0.3819 \\
\text { ns } \\
0.0206 \\
\text { ns }\end{array}$ & $\begin{array}{l}0.4766 \\
\text { ns } \\
0.1423 \\
\text { ns }\end{array}$ \\
\hline
\end{tabular}

MOE, reduced Young's modulus of elasticity; Adhes, adhesion; Deform, deformation; Dissip, energy dissipation

$A$, tracheary element lumen area; $N$, number of tracheary elements per $\mathrm{mm}^{2}$ of the primary xylem area; $F$, tracheary element lumen fraction; $S$, tracheary element size:number ratio;

leaf, leaf thickness; mesoph, mesophyll thickness; palisade, palisade parenchyma thickness; spongy, spongy parenchyma thickness;

height, tree height; BHD, tree diameter at breast height; budburst, spring budburst date; discolor, autumn leaf discolouration date; frost, frost damage; $\mathrm{CO}_{2}$ assi, $\mathrm{CO}_{2}$ assimilation rate; stcond, stomatal conductivity; transpir, transpiration rate; WUE, water-use efficiency; $F_{v} / F_{m}$, the maximum fluorescence yield; $P I$, photosynthetic performance index; $R C A B S$, density of active photosystem II reaction centers $(R C / A B S) ; T_{85}$, thermostability of photosystem II;

long, longitude; lat, latitude; elev, elevation; $B I O 4$, temperature seasonality; $B I O 5$, maximum temperature of the warmest month; BIO6, minimum temperature of the coldest month; $B I O 7$, temperature annual range; $B I O 13$, precipitation of the wettest month; $B I O 14$, precipitation of the driest month; $B I O 15$, precipitation seasonality; $E Q$, Ellenberg 's climatic quotient

variation in tracheary element lumen area and density is shown in Fig. 3. In the northern part of central Europe, a group of provenances can be identified covering eastern Germany, northern Poland and the Czech Republic with small tracheary element lumen areas and high tracheary element densities, while the surrounding provenances display opposite values. Of course, under the absence of statistical significance of differences, such patterns need to be regarded cautiously.

To identify potential associations between structural traits and nanomechanical properties on one side and growth, phenology and physiological traits on the other side (whatever the causal relationships between them may be), linear and quadratic regressions were calculated. Several linear models were significant, while only in one case the quadratic model was significant and, at the same time, performed better than the corresponding linear model (adhesion vs. BIO4); however, non-linearity seems to be due to outliers (data not shown).

Table 3 Kruskal-Wallis tests of inter-provenance differences in leaf nanomechanical, vascular and structural traits

\begin{tabular}{lrrrl}
\hline Variable & DF & \multicolumn{1}{l}{$\chi^{2}$} & $P$ & $R^{2}$ \\
\hline Adhesion & 7 & 13.307 & 0.0650 & 0.6244 \\
Deformation & 7 & 11.320 & 0.1253 & 0.5971 \\
Dissipation & 7 & 6.373 & 0.4970 & 0.3364 \\
Modulus of elasticity & 7 & 12.640 & 0.0814 & 0.5070 \\
Tracheary element lumen area & 14 & 15.180 & 0.3659 & 0.2119 \\
Tracheary element density & 14 & 12.688 & 0.5512 & 0.1902 \\
$F$ & 14 & 20.838 & 0.1059 & 0.3541 \\
$S$ & 14 & 13.828 & 0.4626 & 0.1825 \\
Conductivity & 14 & 12.649 & 0.5543 & 0.2475 \\
Leaf thickness & 14 & 16.528 & 0.2822 & 0.2767 \\
Mesophyll thickness & 14 & 16.874 & 0.2630 & 0.2983 \\
Palisade parenchyma thickness & 14 & 12.516 & 0.5649 & 0.2142 \\
Spongy parenchyma thickness & 14 & 21.218 & 0.0962 & 0.3446 \\
\hline
\end{tabular}

Therefore, a matrix of correlation coefficients (reflecting linear dependence) between these two groups of traits was calculated (Table 2). Surprisingly, no significant correlations were found between vascular traits and water-use-related physiological traits such as stomatal conductance or transpiration rate. Similarly, the correlations between the thickness of leaves and their constituent layers and tree size (height, diameter) were weak and non-significant. Among nanomechanical properties, the modulus of elasticity was found to be higher in later flushing (and thus less frost-damaged) trees, and it was also negatively correlated with tree size (Fig. 4).

At the provenance level, associations of structural and nanomechanical traits with climatic and geographic variables were inspected (Table 2). In this case, more significant correlations were detected. In general, leaf structure traits were significantly correlated with geography and climate: thickness of all leaf layers increased towards the East (Fig. 5a), and also increased with growing temperature seasonality and increasing temperature annual range (Fig. 5d). Vascular traits showed a significant association with latitude (Fig. 5b). Among nanomechanical properties, there is no overall pattern; just the tracheary element cell wall deformation closely correlates with temperature seasonality (Fig. 5c).

\section{Discussion}

\subsection{Correlations among structural traits and nanomechanical properties}

Expectedly, leaf structural traits were closely inter-correlated, just as were vascular traits. On the other hand, correlation patterns among nanomechanical properties of tracheary element cell walls within the primary xylem tissue were less clear. Correlations between leaf traits are in fact trivial and partly due to a mathematical artifact; the internal structure of beech leaves is quite stable (Aranda et al. 2001) and 
Table 4 Means and standard deviations of leaf-anatomical, vascular and nanomechanical cell-wall traits

Provenance

$\begin{aligned} 3 & \text { Picardie } \\ 5 & \text { Bretagne } \\ 30 & \text { Belzig } \\ 35 & \text { Hinterstoder } \\ 36 & \text { Eisenerz } \\ 50 & \text { Janov-Nacetin } \\ 55 & \text { Postojna Javor } \\ 67 & \text { Bilowo }\end{aligned}$

3 Picardie

5 Bretagne

12 Gruenewald

15 Elspeet

23 Torup

26 Farchau

30 Belzig

35 Hinterstoder

36 Eisenerz

39 Jaworze

43 Jawornik

50 Janov-Nacetin

55 Postojna Javor

62 Aarberg

67 Bilowo

(1)

$\begin{aligned} 3 & \text { Picardie } \\ 5 & \text { Bretagne } \\ 12 & \text { Gruenewald } \\ 15 & \text { Elspeet } \\ 23 & \text { Torup } \\ 26 & \text { Farchau } \\ 30 & \text { Belzig } \\ 35 & \text { Hinterstoder } \\ 36 & \text { Eisenerz } \\ 39 & \text { Jaworze } \\ 43 & \text { Jawornik } \\ 50 & \text { Janov-Nacetin } \\ 55 & \text { Postojna Javor } \\ 62 & \text { Aarberg } \\ 67 & \text { Bilowo }\end{aligned}$

$\begin{array}{rr}\text { MOE } & \text { Adhesion } \\ \mathrm{MPa} & \mathrm{nN} \\ 4429.3 \pm 1724.4 & 25.97 \pm 5.60 \\ 3201.0 \pm 885.3 & 17.07 \pm 4.22 \\ 5622.9 \pm 1177.1 & 25.15 \pm 6.58 \\ 7061.2 \pm 1111.1 & 20.01 \pm 3.72 \\ 4517.0 \pm 257.4 & 29.12 \pm 8.85 \\ 3989.0 \pm 648.0 & 20.74 \pm 4.86 \\ 4470.2 \pm 927.7 & 18.98 \pm 5.87 \\ 6313.4 \pm 2993.8 & 39.71 \pm 9.79\end{array}$

Tracheary element area

$$
10^{-4} \mathrm{~mm}^{2}
$$$$
1.05 \pm 0.35
$$

$1.06 \pm 0.01$

$1.10 \pm 0.20$

$0.86 \pm 0.17$

$0.94 \pm 0.17$

$0.92 \pm 0.17$

$0.91 \pm 0.18$

$1.05 \pm 0.12$

$1.05 \pm 0.08$

$1.02 \pm 0.10$

$1.15 \pm 0.10$

$0.82 \pm 0.07$

$1.22 \pm 0.61$

$1.10 \pm 0.13$

$0.99 \pm 0.31$

Leaf thickness $\mu \mathrm{m}$

$158.39 \pm 31.27$

$129.04 \pm 13.38$

$160.37 \pm 25.27$

$136.62 \pm 12.80$

$155.93 \pm 18.80$

$162.02 \pm 26.88$

$148.40 \pm 26.62$

$149.57 \pm 25.03$

$154.10 \pm 13.17$

$170.96 \pm 26.15$

$167.67 \pm 15.62$

$147.46 \pm 19.95$

$163.95 \pm 16.70$

$144.84 \pm 11.00$

$150.15 \pm 7.01$

$370.1 \pm 124.0$
$372.8 \pm 24.1$
$344.5 \pm 48.5$
$365.2 \pm 40.2$
$377.5 \pm 58.5$
$376.8 \pm 84.1$
$455.5 \pm 104.5$
$364.7 \pm 52.5$
$350.7 \pm 51.1$
$409.2 \pm 20.2$
$324.0 \pm 20.3$
$437.4 \pm 60.2$
$366.1 \pm 179.1$
$368.3 \pm 41.8$
$364.7 \pm 64.8$

Nanomechanical cell-wall properties

Deformation

$\mathrm{nm}$

$2.705 \pm 0.223$

$2.181 \pm 0.425$

$2.015 \pm 0.241$

$1.765 \pm 0.093$

$1.987 \pm 0.236$

$2.145 \pm 0.152$

$1.964 \pm 0.219$

$1.855 \pm 0.406$

Vascular traits

Tracheary element density

$F$
$0.0387 \pm 0.0018$
$0.0396 \pm 0.0026$
$0.0380 \pm 0.0026$
$0.0313 \pm 0.0036$
$0.0356 \pm 0.0045$
$0.0345 \pm 0.0019$
$0.0416 \pm 0.0052$
$0.0385 \pm 0.0022$
$0.0367 \pm 0.0048$
$0.0418 \pm 0.0048$
$0.0374 \pm 0.0012$
$0.0361 \pm 0.0035$
$0.0447 \pm 0.0078$
$0.0404 \pm 0.0015$
$0.0360 \pm 0.0046$

\section{F}

Mesophyll thickness

$\mu \mathrm{m}$
$137.31 \pm 29.93$
$107.37 \pm 10.85$
$139.41 \pm 25.09$
$117.49 \pm 11.73$
$135.76 \pm 17.81$
$142.13 \pm 25.20$
$128.34 \pm 25.23$
$130.04 \pm 23.02$
$132.59 \pm 11.72$
$151.47 \pm 26.21$
$147.27 \pm 15.08$
$126.90 \pm 19.38$
$144.95 \pm 17.06$
$125.54 \pm 13.52$
$130.05 \pm 7.15$

Leaf structural traits

Palisade parenchyma thickness

\begin{tabular}{ll}
\multicolumn{1}{c}{$\mu \mathrm{m}$} & $\mu \mathrm{m}$ \\
$84.30 \pm 17.53$ & $53.02 \pm 13.95$ \\
$63.85 \pm 5.63$ & $43.52 \pm 10.21$ \\
$82.84 \pm 17.64$ & $56.57 \pm 11.70$ \\
$76.26 \pm 9.54$ & $41.23 \pm 2.59$ \\
$81.92 \pm 12.54$ & $53.84 \pm 5.98$ \\
$85.32 \pm 13.95$ & $56.80 \pm 11.67$ \\
$78.26 \pm 14.21$ & $50.09 \pm 13.24$ \\
$81.16 \pm 14.11$ & $48.89 \pm 11.19$ \\
$75.60 \pm 11.18$ & $57.00 \pm 4.86$ \\
$88.48 \pm 20.10$ & $63.00 \pm 8.60$ \\
$88.56 \pm 14.58$ & $58.71 \pm 6.48$ \\
$75.97 \pm 15.28$ & $50.93 \pm 7.87$ \\
$84.98 \pm 10.41$ & $59.98 \pm 11.78$ \\
$79.60 \pm 8.68$ & $45.94 \pm 4.98$ \\
$78.34 \pm 2.43$ & $51.71 \pm 8.60$
\end{tabular}

Energy dissipation

$\mathrm{eV}$

$1407.4 \pm 750.5$

$1119.5 \pm 593.9$

$1157.5 \pm 877.4$

$733.4 \pm 139.1$

$1688.8 \pm 1311.6$

$1486.6 \pm 159.8$

$936.2 \pm 629.2$

$2242.7 \pm 901.8$

$\begin{array}{cc}S & \text { Relative xylem conductivity } \\ 10^{-7} \mathrm{~mm}^{4} & 10^{-7}{\mu \mathrm{m}^{4} \mathrm{~mm}^{-2}}_{2} \\ 2.83 \pm 2.13 & 1.167 \pm 0.424 \\ 2.85 \pm 0.20 & 1.467 \pm 0.229 \\ 3.21 \pm 1.06 & 1.362 \pm 0.360 \\ 2.34 \pm 0.70 & 0.918 \pm 0.403 \\ 2.50 \pm 0.88 & 1.068 \pm 0.315 \\ 2.43 \pm 0.90 & 1.052 \pm 0.294 \\ 2.01 \pm 1.07 & 1.292 \pm 0.416 \\ 2.89 \pm 0.85 & 1.403 \pm 0.061 \\ 2.99 \pm 0.50 & 1.354 \pm 0.291 \\ 2.50 \pm 0.27 & 1.420 \pm 0.484 \\ 3.56 \pm 0.54 & 1.368 \pm 0.316 \\ 1.89 \pm 0.37 & 0.995 \pm 0.144 \\ 3.33 \pm 6.42 & 1.410 \pm 0.402 \\ 2.98 \pm 0.70 & 1.412 \pm 0.456 \\ 2.71 \pm 1.43 & 1.205 \pm 0.666\end{array}$

$M O E$, reduced Young's modulus of elasticity; $F$, tracheary element lumen fraction; $S$, tracheary element size:number ratio 

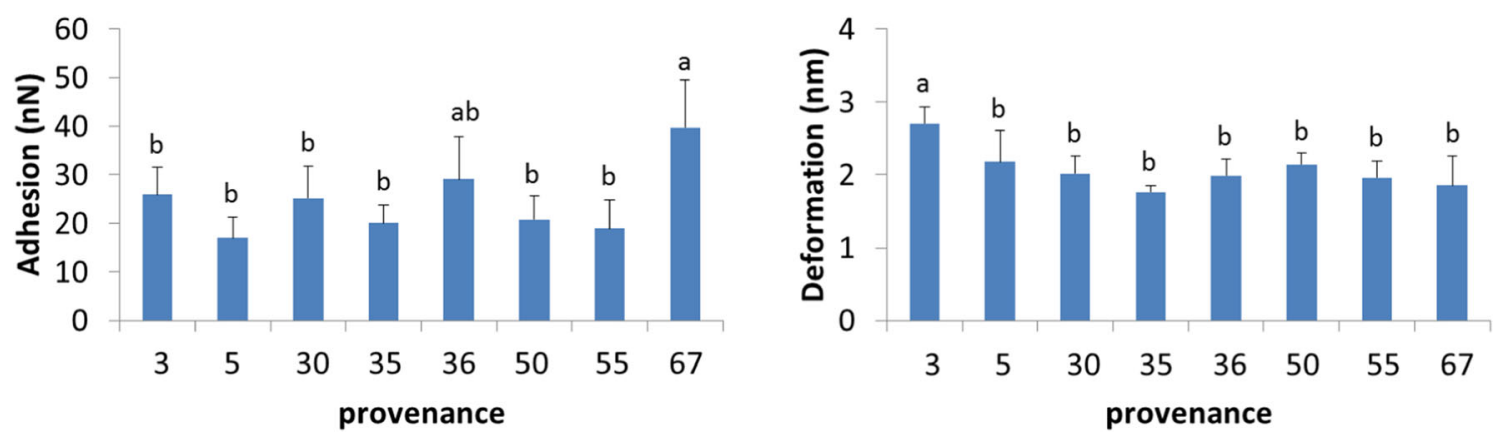

Fig. 2 Provenance means (+ standard deviations) of nanomechanical properties (adhesion; left, and deformation; right) in beech provenances. Differences between means denoted by the same letter are non-significant (pairwise Wilcoxon test)

thicknesses of individual layers add up to leaf thickness. The same partly applies to vascular traits, where again the parameters are partly mathematically related.

The relationships between the vascular properties of midrib veins and leaf tissue thickness were much looser. Generally, thicker leaves (especially those with a thicker spongy parenchyma) tended to have larger and less numerous tracheary elements. The diameter of midrib veins probably correlates with the size of secondary and higher-order veins distributing water in the leaf lamina (Coomes et al. 2008; Gleason et al. 2018), as lateral conduction of water through the palisade parenchyma is limited, and a higher proportion of secondary veins in the lamina may contribute to a shorter water diffusion pathway from the vein endings to the mesophyll cells (CastroDíez et al. 2000; Sack and Holbrook 2006). In the Fagaceae, having a high proportion of sclerified tissues, which consist primarily of the sclerenchyma of minor veins (Cornelissen et al. 1996), a higher diameter of midrib and secondary veins may result in a better leaf growth.

On the other hand, there were found non-significant trait linkages between the nanomechanical properties of tracheary element cell walls and the leaf midrib structure. This result is quite opposite to that found for leaves of clonally micropropagated Dutch elm interspecific hybrids
'Groeneveld' and 'Dodoens' when MOE negatively correlated with several traits such as theoretical relative conductivity, tracheary element lumen area, tracheary element size to number ratio, and also positively correlated with the number of tracheary elements per unit area (Durkovič et al. 2013). In the wall of a living cell, there is a feedback between mechanical stresses imposed by growth and cell physiology and the cellular machinery involved in the deposition of macromolecules determining nanomechanical properties of a cell wall. However, xylem cells are dead. Consequently, there is a potential for post-mortem processes modifying cell wall properties. Cell wall is a chemically heterogeneous material, where distinct molecular domains within a wall exhibit different nanomechanical properties (Farahi et al. 2017). From this viewpoint, geographical variation in the chemical composition of beech xylem cell walls might be responsible for the lack of associations between cell wall nanomechanical properties and midrib vascular traits. Janota and Kurjatko (1978) found a very low geographical variation in wood density of beech trees across Slovakia. Wood density is one of the key traits that seems to encapsulate, if not link, many of the plant functional traits (Swenson and Enquist 2007), including the distributions of vessel sizes and densities and also the
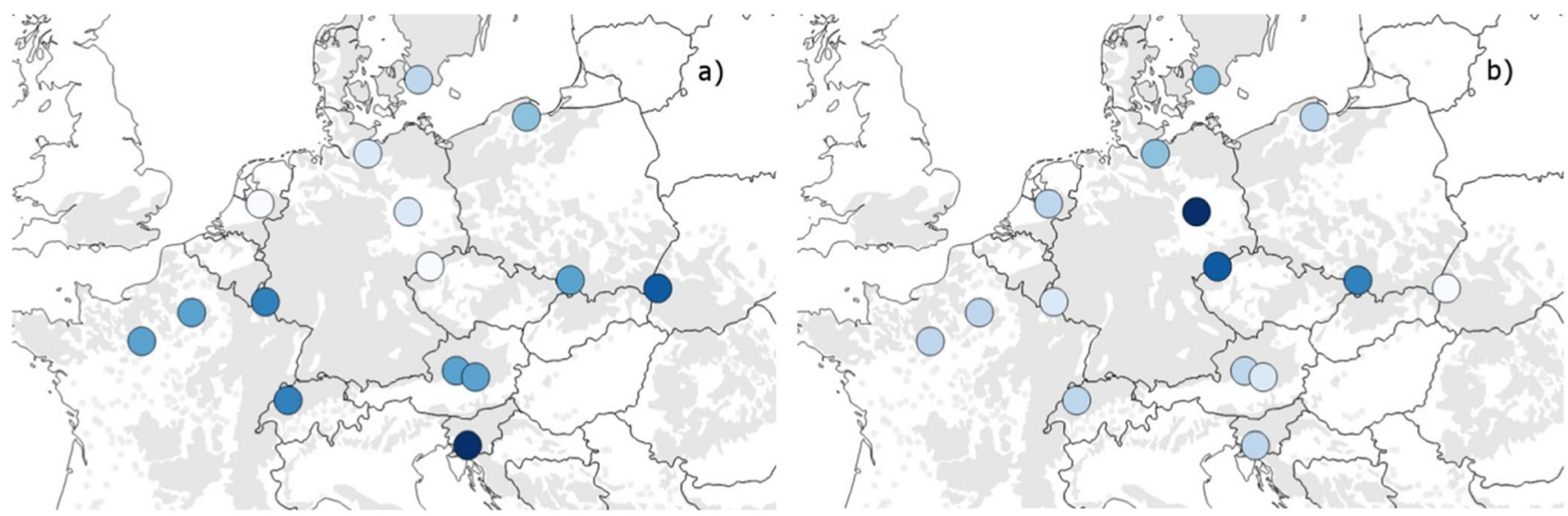

Fig. 3 Tracheary element lumen area (left) and tracheary element density (right). Darker symbol colours mean higher average values of the respective trait 

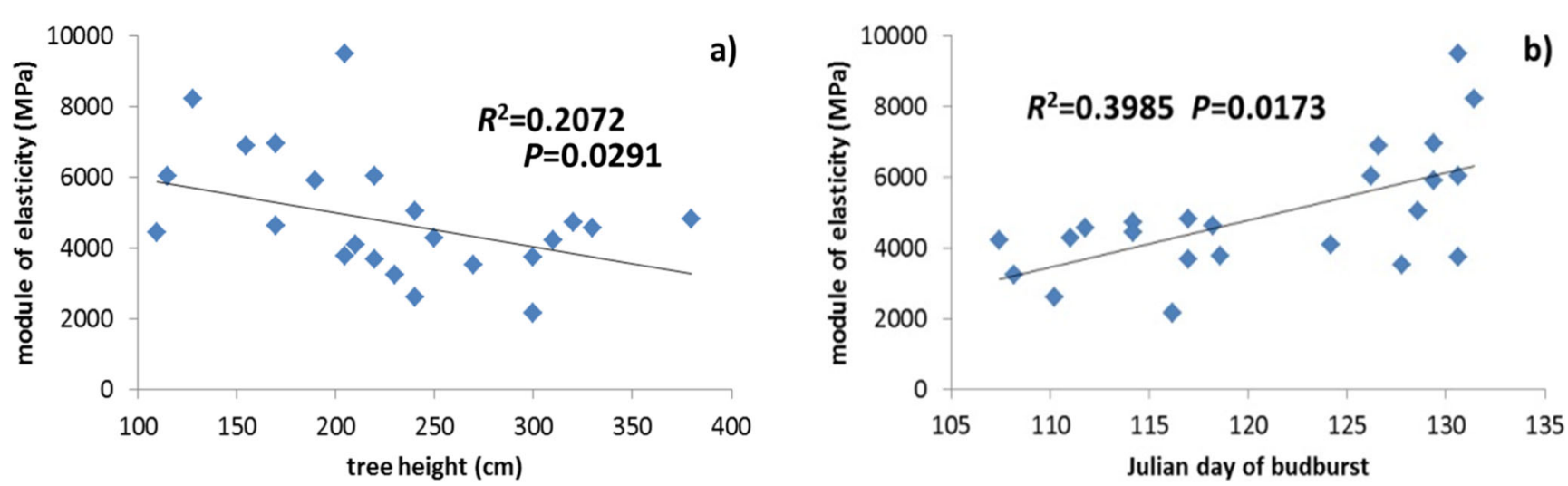

Fig. 4 Relationships between the module of elasticity and tree height (a) and budburst date (b)

mechanical properties of xylem tissue such as cell wall stiffness quantified by MOE and cell wall toughness quantified by energy dissipation.

\subsection{Associations with growth, physiology and climate}

As both vascular and leaf anatomical traits were largely intercorrelated, they showed generally consistent responses to geography and climatic variables; however, significant responses were scarce, and their interpretation in terms of climatic adaptation is not straightforward. Leaf anatomy is obviously a plastic trait, very responsive to site conditions (Stojnić et al. 2016). It often shows clear geographic patterns and
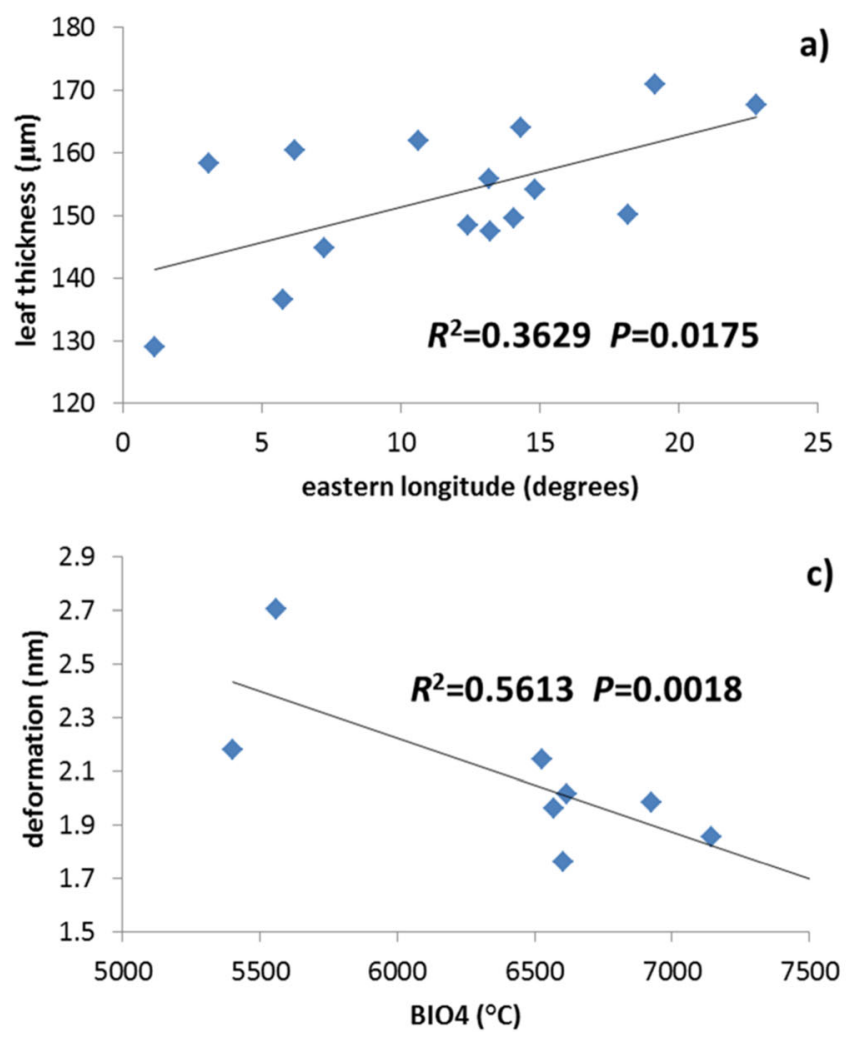

climatic trends when measured in situ (Bussotti et al. 1998, 2005) but such trends represent more likely results of acclimation to local levels of water stress (Bussotti et al. 1995; Gravano et al. 1999) than heritable adaptations. Consequently, in spite of a provenance effect present in the variation of most traits, a strong genotype-by-environment interaction obscures geographical and climatic trends in terms of the site of origin (Stojnić et al. 2016). Similarly, vascular properties equally react on site conditions, and geographical trends also result primarily from acclimation (Sanginés de Cárcer et al. 2017).

In spite of this, several rangewide trends related to the site of origin and associations with phenotype were identified;
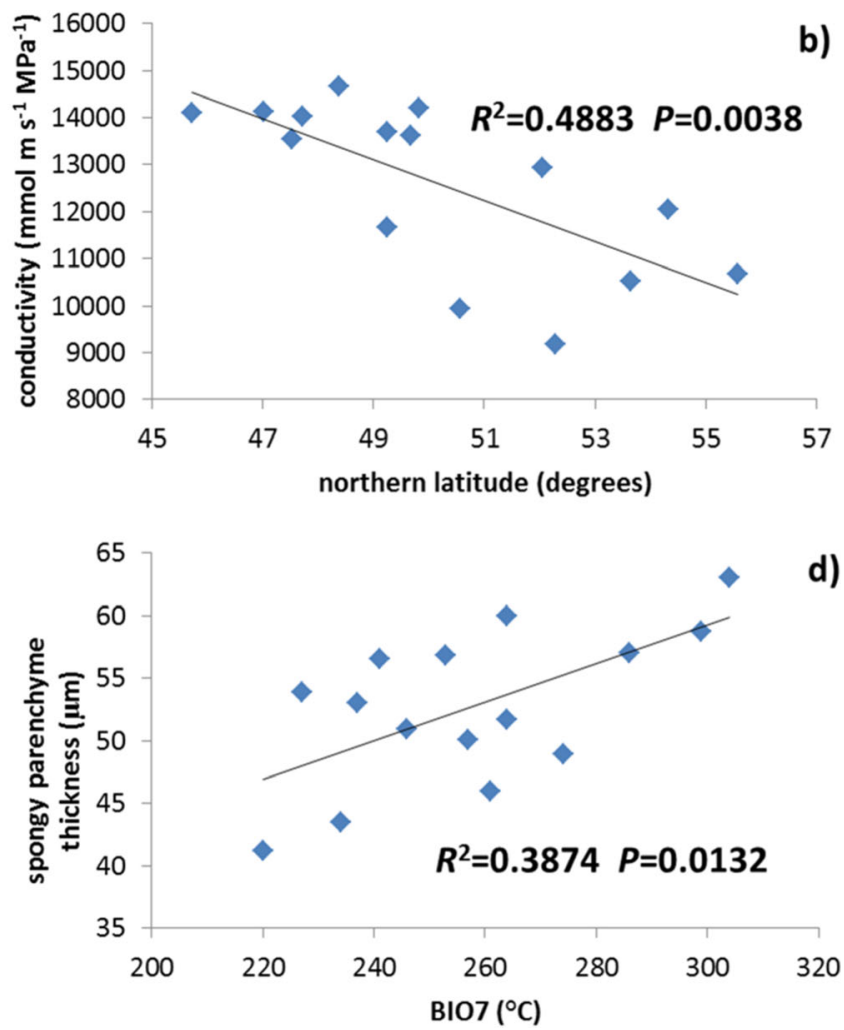

Fig. 5 Relationships between leaf, vascular and cell-wall properties and geographical and climatic variables 
however, there is little correspondence between the correlations on the population level (geography and climate) and individual level (phenotypic traits). Leaf parenchyma traits were found to be correlated with longitude. At the same time, they also showed significant associations with the proxies of climate continentality such as temperature seasonality or temperature annual range. The true driver of such relationships remains unknown, however. Another trait displaying a very clear West-East trend is vegetative phenology, especially budburst date, which decreases towards the East (von Wuehlisch et al. 1995). Associations between leaf anatomy and vegetative phenology were mostly found at the species level (Panchen et al. 2014; Osada 2017; Gougherty and Gougherty 2018), but they exist also on the population level (Zhong et al. 2018). Leaf structure is strongly affected by irradiation levels during leaf development (Oguchi et al. 2005), which means that different budburst dates may have direct influence on leaf anatomy. Therefore, we expected that phenology may explain the observed climatic correlations also in our study. However, on the individual level, no significant correlations between leaf structure and phenological traits were observed.

Like leaf parenchyma thickness, leaf venation characteristics frequently display geographic and climatic trends when measured in situ (Sanginés de Cárcer et al. 2017; Schneider et al. 2018) but the importance of the heritable component of this variation is largely unknown. In our study, vascular properties of leaf midribs showed a latitudinal trend: decreasing tracheary element lumen area and relative conductivity towards the North. As responses of leaf xylem traits to temperature and precipitation variables were non-significant, photoperiod remains as the sole latitude-associated factor, which may have influenced properties of tracheary elements. Again, photoperiod is an important driver of leaf and cambium phenology (Falusi and Calamassi 1990) but the absence of associations between leaf midrib properties and phenology on the individual level renders this explanation implausible. In contrast, significant correlations of the cell wall modulus of elasticity were found with phenology and/or growth, but not with climate or geography. Cell wall nanomechanical properties are known to be formed primarily during the growth phase and later react on changes of turgor in a limited extent and with a certain delay (Milani et al. 2013; Cosgrove 2016). During the plant cell growth, the cell wall is deformed in response to turgor pressure, and usually new material is added to the cell wall. However, although elasticity and growth are correlated, the causal relationship between cell wall elasticity, viscoelasticity, turgor pressure and growth is still unclear (Derbyshire et al. 2007; Routier-Kierzkowska and Smith 2013). Timing of cell growth is thus relevant: temperatures, eventual occurrence of frosts, water availability etc. - all these factors change during the budburst season in the spring, and by affecting the speed of cell division and cell growth they indirectly may influence the course of cell wall formation.

\section{Conclusion}

The studied leaf-structural properties exhibited rangewide trends and associations with climatic gradients. In spite of an inconsistency among the structural levels (leaf, midrib, cell walls), their existence indicates underlying adaptive mechanisms. Just to remind: the advantage of common-garden experiments such as provenance tests is that they largely eliminate environment-driven phenotypic variation among the tested populations. The observed differences among provenances and their association with climate or latitude can thus be attributed to long-term adaptation of their genetic structures to the environments at their sites of origin. In addition to adaptation, neutral processes may have contributed to the observed geographical patterns. We tried to eliminate their influence by limiting our study to populations, which all have originated from a single glacial refugium located in the Eastern Alps or Istria. In spite of this, changes of genetic structures during the Holocene colonization (e.g., a gradual loss of alleles towards the range margins due to recurrent founder effect; Comps et al. 2001) may also be partly responsible for geographical trends. Our study indicates that leaf structural traits (relevant in terms of water economy of a tree) are associated with the continentality of the climate, which is expected to increase with ongoing climate change and poses a serious threat to mesic temperate hardwoods such as European beech. Although the spatial resolution of our study is too coarse for practical application, it indicates that leaf structural traits may be useful indicators to guide assisted migration, i.e. transfer of forest reproductive materials aimed at adapting future forests to changed climate.

Acknowledgements Technical assistance of Ingrid Čaňová, Lucia Javoříková and Michal Moravčík with sample preparation for light microscopy is greatly appreciated. The authors also thank to Elizabeth Ritch-Krč for language revision.

Funding information This study was funded by the Slovak Grant Agency for Science, grant numbers VEGA 1/0450/19 and VEGA $1 / 0029 / 20$.

Data availability The datasets generated during the current study are available as open-access data in the Zenodo repository at https://doi.org/ 10.5281/zenodo. 3827130 .

\section{Compliance with ethical standards}

Conflict of interest The authors declare that they have no conflict of interests. 


\section{References}

Aasamaa K, Niinemets U, Sober A (2005) Leaf hydraulic conductance in relation to anatomical and functional traits during Populus tremula leaf ontogeny. Tree Physiol 25:1409-1418. https://doi.org/10.1093/ treephys/25.11.1409

Allen CD, Macalady AK, Chenchouni H, Bachelet D, Mcdowell N, Vennetier M, Kitzberger T, Rigling A, Breshears DD, Hogg EH, Gonzalez P, Fensham R, Zhang Z, Castro J, Demidova N, Lim J-H, Allard G, Running SW, Semerci A, Cobb N (2010) A global overview of drought and heat-induced tree mortality reveals emerging climate change risks for forests. For Ecol Manage 259:660-684. https://doi.org/10.1016/j.foreco.2009.09.001

Aranda I, Bergasa LF, Gil L, Pardos JA (2001) Effects of relative irradiance on the leaf structure of Fagus sylvatica L. seedlings planted in the understory of a Pinus sylvestris L. stand after thinning. Ann For Sci 58:673-680

Arslan B, Ju X, Zhang X, Abu-Lail NI (2015) Heterogeneity and specificity of nanoscale adhesion forces measured between selfassembled monolayers and lignocellulosic substrates: a chemical force microscopy study. Langmuir 31:10233-10245

Bréda N, Huc R, Granier A, Dreyer E (2006) Temperate forest trees and stands under severe drought: a review of ecophysiological responses, adaptation processes and long-term consequences. Ann For Sci 63:625-644. https://doi.org/10.1051/forest:2006042

Brestič M, Živčák M (2013) PSII fluorescence techniques for measurement of drought and high temperature stress signal in crop plants: protocols and applications. In: Rout GR, Das AB (eds) Molecular Stress Physiology of Plants. Springer India, India, pp 87-131

Brodribb TJ, Feild TS (2010) Leaf hydraulic evolution led a surge in leaf photosynthetic capacity during early angiosperm diversification. Ecol Lett 13:175-183. https://doi.org/10.1111/j.1461-0248.2009. 01410.x

Brodribb TJ, Holbrook NM, Zwieniecki MA, Palma B (2005) Leaf hydraulic capacity in ferns, conifers and angiosperms: impacts on photosynthetic maxima. New Phytol 165:839-846. https://doi.org/10. 1111/j.1469-8137.2004.01259.x

Buckley TN, John GP, Scoffoni C, Sack L (2015) How does leaf anatomy influence water transport outside the xylem? Plant Physiol 168: 1616-1635. https://doi.org/10.1104/pp.15.00731

Bussotti F, Bottacci A, Bartolesi A, Grossoni P, Tani C (1995) Morphoanatomical alterations in leaves collected from beech trees (Fagus sylvatica L.) in conditions of natural water stress. Environ Exp Bot 35:201-213. https://doi.org/10.1016/0098-8472(94)00040-C

Bussotti F, Gravano E, Grossoni P, Tani C (1998) Occurrence of tannins in leaves of beech trees (Fagus sylvatica L.) along an ecological gradient, detected with histochemical and ultrastructural analyses. New Phytol 138:469-479. https://doi.org/10.1046/j.1469-8137. 1998.00121.x

Bussotti F, Pancrazi M, Matteucci G, Gerosa G (2005) Leaf morphology and chemistry in Fagus sylvatica (beech) trees as affected by site factors and ozone: results from CONECOFOR permanent monitoring plots in Italy. Tree Physiol 25:211-219. https://oi.org/10.1093/ treephys/25.2.211

Castro-Díez P, Puyravaud JP, Cornelissen JHC (2000) Leaf structure and anatomy as related to leaf mass per area variation in seedlings of a wide range of woody plant species and types. Oecologia 124:476486. https://doi.org/10.1007/PL00008873

Choat B, Brodribb TJ, Brodersen CR, Duursma RA, Lopez R, Medlyn BE (2018) Triggers of tree mortality under drought. Nature 558: 531-539. https://doi.org/10.1038/s41586-018-0240-x

Clausen CA (2010) Biodeterioration of wood. In: wood handbookwood as an engineering material. General Technical Report FPLGTR-190, Centennial edn. US Department of Agriculture, Forest Service, Madison, WI, pp. 14-1-14-16
Comps B, Gömöry D, Letouzey J, Thiébaut B, Petit RJ (2001) Diverging trends between heterozygosity and allelic richness during postglacial colonization in the European beech. Genetics 157:389-397

Coomes DA, Heathcote S, Godfrey ER, Shepherd JJ, Sack L (2008) Scaling of xylem vessels and veins within the leaves of oak species. Biol Lett 4:302-306. https://doi.org/10.1098/rsbl.2008.0094

Cornelissen JHC, Castro Diez P, Hunt R (1996) Seedling growth, allocation and leaf attributes in a wide range of woody plant species and types. J Ecol 84:755-765. https://doi.org/10.2307/2261337

Cosgrove DJ (2016) Plant cell wall extensibility: connecting plant cell growth with cell wall structure, mechanics, and the action of wallmodifying enzymes. J Exp Bot 67:463-476. https://doi.org/10. 1093/jxb/erv511

Czucz B, Galhidy L, Mátyás C (2011) Present and forecasted xeric climatic limits of beech and sessile oak distribution at low altitudes in Central Europe. Ann For Sci 68:99-108. https://doi.org/10.1007/ s13595-011-0011-4

Derbyshire P, Findlay K, McCann MC, Roberts K (2007) Cell elongation in Arabidopsis hypocotyls involves dynamic changes in cell wall thickness. J Exp Bot 58:2079-2089. https://doi.org/10.1093/jxb/ erm074

Dufrêne YF, Martínez-Martín D, Medalsy I, Alsteens D, Müller DJ (2013) Multiparametric imaging of biological systems by forcedistance curve-based AFM. Nat Methods 10:847-854

Duurkovič J, Kardošová M (2020) Leaf anatomy, vascular traits and nanomechanical cell-wall properties in European beech provenances. [dataset]. Zenodo repository. V2. https://doi.org/10.5281/zenodo. 3827130

Ďurkovič J, Čaňová I, Lagaňa R, Kučerová V, Moravčík M, Priwitzer T, Urban J, Dvořák M, Krajňáková J (2013) Leaf trait dissimilarities between Dutch elm hybrids with a contrasting tolerance to Dutch elm disease. Ann Bot 111:215-227. https://doi.org/10.1093/aob/ $\operatorname{mcs} 274$

Ďurkovič J, Husárová H, Javoř́íková L, Čaňová I, Šuleková M, Kardošová M, Lukáčik I, Mamoňová M, Lagaňa R (2017) Physiological, vascular and nanomechanical assessment of hybrid poplar leaf traits in micropropagated plants and plants propagated from root cuttings: a contribution to breeding programs. Plant Physiol Biochem 118:449-459

Falusi M, Calamassi R (1990) Bud dormancy in beech (Fagus sylvatica L.) - effect of chilling and photoperiod on dormancy release of beech seedlings. Tree Physiol 6:429-438. https://doi.org/10.1093/ treephys/6.4.429

Farahi RH, Charrier AM, Tolbert A, Lereu AL, Ragauskas A, Davison BH, Passian A (2017) Plasticity, elasticity, and adhesion energy of plant cell walls: nanometrology of lignin loss using atomic force microscopy. Sci Rep-UK 7:152. https://doi.org/10.1038/s41598017-00234-4

Fletcher LR, Cui HX, Callahan H, Scoffoni C, John GP, Bartlett MK, Burge DO, Sack L (2018) Evolution of leaf structure and drought tolerance in species of Californian Ceanothus. Am J Bot 105:16721687. https://doi.org/10.1002/ajb2.1164

Froux F, Ducrey M, Epron D, Dreyer E (2004) Seasonal variations and acclimation potential of the thermostability of photochemistry in four Mediterranean conifers. Ann For Sci 61:235-241

Gleason SM, Blackman CJ, Gleason ST, McCulloh KA, Ocheltree TW, Westoby M (2018) Vessel scaling in evergreen angiosperm leaves con-forms with Murray's law and area-filling assumptions: implications for plant size, leaf size and cold tolerance. New Phytol 218: 1360-1370. https://doi.org/10.1111/nph.15116

Gömöry D, Ditmarová L', Hrivnák M, Jamnická G, Kmet' J, Krajmerová D, Kurjak D (2015) Differentiation in phenological and physiological traits in European beech (Fagus sylvatica L.). Eur J For Res 134: 1075-1085. https://doi.org/10.1007/s10342-015-0910-2 
Gömöry D, Paule L (2011) Trade-off between height growth and spring flushing in common beech (Fagus sylvatica L.). Ann For Sci 68: 975-984. https://doi.org/10.1007/s13595-011-0103-1

Gömöry D, Paule L, Vyšný J (2007) Patterns of allozyme variation in western Eurasian Fagus. Bot J Linn Soc 154:165-174. https://doi. org/10.1111/j.1095-8339.2007.00666.x

Gougherty AV, Gougherty SW (2018) Sequence of flower and leaf emergence in deciduous trees is linked to ecological traits, phylogenetics, and climate. New Phytol 220:121-131. https://doi.org/10.1111/nph. 15270

Gravano E, Bussotti F, Grossoni P, Tani C (1999) Morpho-anatomical and functional modifications in beech leaves on the top ridge of the Apennines (central Italy). Phyton-Ann Rei Bot A 39:41-46

Guerra FP, Richards JH, Fiehn O, Famula R, Stanton BJ, Shuren R, Sykes R, Davis MF, Neale DB (2016) Analysis of the genetic variation in growth, ecophysiology, and chemical and metabolomic composition of wood of Populus trichocarpa provenances. Tree Genet Genomes 12:6. https://doi.org/10.1007/s11295-015-0965-8

Hartmann H (2015) Carbon starvation during drought-induced tree mortality - are we chasing a myth? J Plant Hydraul 2:e005. https://doi. org/10.20870/jph.2015.e005

Hijmans RJ, Cameron SE, Parra JL, Jones PG, Jarvis A (2005) Very high resolution interpolated climate surfaces for global land areas. Int $\mathrm{J}$ Clim 25:1965-1978. https://doi.org/10.1002/joc.1276

IPCC (2014) Climate change 2014: synthesis report. In: Core Writing Team, Pachauri RJ, Meyer LA (eds.) Contribution of Working Groups I, II and III to the fifth assessment report of the Intergovernmental Panel on Climate Change. IPCC, Geneva, $151 \mathrm{pp}$

Janmey PA, Winer JP, Murray ME, Wen Q (2009) The hard life of soft cells. Cell Motil Cytoskeleton 66:597-605

Janota I, Kurjatko S (1978) Premenlivost' hustoty bukového dreva. Drevársky výskum 23:25-40

Lommel M, Winterhalter PR, Willer T, Dahlhoff M, Schneider MR, Bartels MF, Renner-Müller I, Ruppert T, Wolf E, Strahl S (2013) Protein O-mannosylation is crucial for E-cadherin-mediated cell adhesion. Proc Natl Acad Sci U S A 110:21024-21029

Magri D, Vendramin GG, Comps B, Dupanloup I, Geburek T, Gömöry D, Latalowa M, Litt T, Paule L, Roure JM, Tantau I, van der Knaap WO, Petit RJ, de Beaulieu J-L (2006) Palaeobotanical and genetic data outline the Quaternary history of European beech populations. New Phytol 171:199-222. https://doi.org/10.1111/j.1469-8137. 2006.01740.x

McDowell N, Pockman WT, Allen CD, Breshears DD, Cobb N, Kolb T, Plaut J, Sperry J, West A, Williams DG, Yepez EA (2008) Mechanisms of plant survival and mortality during drought: why do some plants survive while others succumb to drought? New Phytol 178:719-739. https://doi.org/10.1111/j.1469-8137.2008. 02436.x

Milani P, Braybrook SA, Boudaoud A (2013) Shrinking the hammer: micromechanical approaches to morphogenesis. J Exp Bot 64: 4651-4662. https://doi.org/10.1093/jxb/ert169

Nadal M, Flexas J, Gulias J (2018) Possible link between photosynthesis and leaf modulus of elasticity among vascular plants: a new player in leaf traits relationships? Ecol Lett 21:1372-1379. https://doi.org/10. 1111/ele.13103

Nardini A, Luglio J (2014) Leaf hydraulic capacity and drought vulnerability: possible trade-offs and correlations with climate across three major biomes. Funct Ecol 28:810-818. https://doi.org/10.1111/ 1365-2435.12246

Nardini A, Peda G, La Rocca N (2012) Trade-offs between leaf hydraulic capacity and drought vulnerability: morpho-anatomical bases, carbon costs and ecological consequences. New Phytol 196:788-798. https://doi.org/10.1111/j.1469-8137.2012.04294.x

Oguchi R, Hikosaka K, Hirose T (2005) Leaf anatomy as a constraint for photosynthetic acclimation: differential responses in leaf anatomy to increasing growth irradiance among three deciduous trees. Plant
Cell Environ 28:916-927. https://doi.org/10.1111/j.1365-3040. 2005.01344.x

Osada N (2017) Relationships between the timing of budburst, plant traits, and distribution of 24 coexisting woody species in a warmtemperate forest in Japan. Am J Bot 104:550-558. https://doi.org/ 10.3732/ajb.1600444

Panchen ZA, Primack RB, Nordt B, Ellwood ER, Stevens AD, Renner SS, Willis CG, Fahey R, Whittemore A, Du YJ, Davis CC (2014) Leaf out times of temperate woody plants are related to phylogeny, deciduousness, growth habit and wood anatomy. New Phytol 203: 1208-1219. https://doi.org/10.1111/nph.12892

Poljanec A, Ficko A, Bončina A (2010) Spatiotemporal dynamic of European beech (Fagus sylvatica L.) in Slovenia, 1970-2005. For Ecol Manage 259:2183-2190. https://doi.org/10.1016/j.foreco. 2009.09.022

Ren D, Wang H, Yu Z, Wang H, Yu Y (2015) Mechanical imaging of bamboo fiber cell walls and their composites by means of peakforce quantitative nanomechanics (PQNM) technique. Holzforschung 69: 975-984

Rennenberg H, Seiler W, Matyssek R, Gessler A, Kreuzwieser J (2004) European beech (Fagus sylvatica L.) - a forest tree without future in the south of Central Europe? Allg Forst Jagdztg 175:210-224

Routier-Kierzkowska A-L, Smith RS (2013) Measuring the mechanics of morphogenesis. Curr Opin Plant Biol 16:25-32. https://doi.org/10. 1016/j.pbi.2012.11.002

Sack L, Frole K (2006) Leaf structural diversity is related to hydraulic capacity in tropical rain forest trees. Ecology 87:483-491. https:// doi.org/10.1890/05-0710

Sack L, Holbrook NM (2006) Leaf hydraulics. Annu Rev Plant Biol 57: 361-381. https://doi.org/10.1146/annurev.arplant.56.032604. 144141

Sala A, Piper F, Hoch G (2010) Physiological mechanisms of droughtinduced tree mortality are far from being resolved. New Phytol 186: 274-281. https://doi.org/10.1111/j.1469-8137.2009.03167.x

Sanginés de Cárcer P, Signarbieux C, Schlaepfer R, Buttler A, Vollenweider P (2017) Responses of antinomic foliar traits to experimental climate forcing in beech and spruce saplings. Environ Exp Bot 140:128-140. https://doi.org/10.1016/j.envexpbot.2017. 05.013

SAS (2010) SAS® STAT User's Guide. http://support.sas.com/ documentation/onlinedoc/91pdf/sasdoc_91/stat_ug_7313.pdf. Accessed 14 Feb 2010

Schneider JV, Negraschis V, Habersetzer J, Rabenstein R, Wesenberg J, Wesche K, Zizka G (2018) Taxonomic diversity masks leaf veinclimate relationships: lessons from herbarium collections across a latitudinal rainfall gradient in West Africa. Bot Lett 165:384-395. https://doi.org/10.1080/23818107.2017.1421480

Scoffoni C, Pou A, Aasamaa K, Sack L (2008) The rapid light response of leaf hydraulic conductance: new evidence from two experimental methods. Plant Cell Environ 31:1803-1812. https://doi.org/10. 1111/j.1365-3040.2008.01884.x

Sellin A, Kupper P (2007) Temperature, light and leaf hydraulic conductance of little-leaf linden (Tilia cordata) in a mixed forest canopy. Tree Physiol 27:679-688. https://doi.org/10.1093/treephys/27.5. 679

Stackpole DJ, Vaillancourt RE, Alves A, Rodrigues J, Potts BM (2011) Genetic variation in the chemical components of Eucalyptus globulus wood. G3-Genes Genom Genet 1:151-159. https://doi. org/10.1534/g3.111.000372

Stojnić S, Orlović S, Miljković D, von Wuehlisch G (2016) Intra- and interprovenance variations in leaf morphometric traits in European beech (Fagus sylvatica L.). Arch Biol Sci 68:781-788. https://doi. org/10.2298/ABS151008064S

Stojnić S, Suchocka M, Benito-Garzon M, Torres-Ruiz JM, Cochard H, Bolte A, Cocozza C, Cvjetković B, de Luis M, Martinez-Vilalta J, Raebild A, Tognetti R, Delzon S (2018) Variation in xylem 
vulnerability to embolism in European beech from geographically marginal populations. Tree Physiol 38:173-185. https://doi.org/10. 1093/treephys/tpx128

Strasser RJ, Srivastava A, Tsimilli-Michael M (2000) The fluorescence transient as a tool to characterize and screen photosynthetic samples. In: Yunus M, Pathre U, Mohanty P (eds) Probing photosynthesis: Mechanisms, regulation and adaptation. Taylor \& Francis, London, pp 445-483.

Swenson NG, Enquist BJ (2007) Ecological and evolutionary determinants of a key plant functional trait: wood density and its community-wide variation across latitude and elevation. Am J Bot 94:451-459. https://doi.org/10.3732/ajb.94.3.451

von Wuehlisch G, Krusche D, Muhs HJ (1995) Variation in temperature sum requirement for flushing of beech provenances. Silvae Genet 44:5-6

Yang S, Tyree MT (1994) Hydraulic architecture of Acer saccharum and A. rubrum: comparison of branches to whole trees and the contribution of leaves to hydraulic resistance. J Exp Bot 45:179-186. https:// doi.org/10.1093/jxb/45.2.179
Zanne AE, Westoby M, Falster DS, Ackerly DD, Loarie SR, Arnold SEJ, Coomes DA (2010) Angiosperm wood structure: global patterns in vessel anatomy and their relation to wood density and potential conductivity. Am J Bot 97:207-215. https://doi.org/10.3732/ajb. 0900178

Zhang YJ, Rockwell FE, Wheeler JK, Holbrook NM (2014) Reversible deformation of transfusion tracheids in Taxus baccata is associated with a reversible decrease in leaf hydraulic conductance. Plant Physiol 165:1557-1565. https://doi.org/10.1104/pp.114.243105

Zhong MY, Shao XQ, Wu RX, Wei XT, van Logtestijn RSP, Cornelissen JHC (2018) Contrasting altitudinal trends in leaf anatomy between three dominant species in an alpine meadow. Aust J Bot 66:448458. https://doi.org/10.1071/BT17247

Zimmermann MH (1983) Xylem structure and the ascent of sap. Springer-Verlag, Berlin

Publisher's note Springer Nature remains neutral with regard to jurisdictional claims in published maps and institutional affiliations. 